\title{
WILEY-VCH
}

\section{Impact of tin fluoride additive on the properties of mixed tin-lead iodide perovskite}

semiconductors

Kimberley J. Savill, Aleksander M. Ulatowski, Michael D. Farrar, Michael B. Johnston, Henry J. Snaith, and Laura M. Herz*

K. J. Savill, A. M. Ulatowski, M. D. Farrar, Prof. M. J. Johnston, Prof. H. J. Snaith, Prof. L.M. Herz

Department of Physics, University of Oxford, Clarendon Laboratory, Parks Road, Oxford OX1 3PU, U.K.

E-mail: laura.herz@physics.ox.ac.uk

Keywords: lead-tin perovskites, photovoltaics, $\mathrm{SnF}_{2}$, charge-carrier dynamics, lattice vibrations

Mixed tin-lead halide perovskites are promising low-band-gap absorbers for all-perovskite tandem solar cells that offer higher efficiencies than single-junction devices. A significant barrier to higher performance and stability is the ready oxidation of tin, commonly mitigated by various additives whose impact is still poorly understood for mixed tin-lead perovskites. Here, the effects of the commonly used $\mathrm{SnF}_{2}$ additive are revealed for $\mathrm{FA}_{0.83} \mathrm{Cs}_{0.17} \mathrm{Sn}_{x} \mathrm{~Pb}_{1-x} \mathrm{I}_{3}$ perovskites across the full compositional lead-tin range and $\mathrm{SnF}_{2}$ percentages of $0.1-20 \%$ of precursor tin content. $\mathrm{SnF}_{2}$ addition causes a significant reduction in the background hole density associated with tin vacancies, yielding longer photoluminescence lifetimes, decreased energetic disorder, reduced Burstein-Moss shifts, and higher charge-carrier mobilities. Such effects are optimized for $\mathrm{SnF}_{2}$ addition of $1 \%$, while for $5 \% \mathrm{SnF}_{2}$ and above, additional nonradiative recombination pathways begin to appear. It is further found that the addition of $\mathrm{SnF}_{2}$ reduces a tetragonal distortion in the perovskite structure deriving from the presence of tin vacancies that cause strain, particularly for high tin content. The optical phonon response associated with inorganic lattice vibrations is further explored, exhibiting a shift to higher frequency and significant broadening with increasing tin fraction, in accordance with lower effective atomic metal masses and shorter phonon lifetimes. 


\section{WILEY-VCH}

\section{Introduction}

Hybrid metal-halide perovskite semiconductors display many properties beneficial for solar cell applications, including large absorption coefficients, ${ }^{[1]}$ low exciton binding energies, ${ }^{[2,3]}$ low trap densities with energetically shallow traps, ${ }^{[4,5]}$ as well as high mobilities and long diffusion lengths of photoexcited charge carriers. ${ }^{[6,7]}$ By virtue of these properties, the power conversion efficiency (PCE) of perovskite solar cells has continued to rise in recent years, currently exceeding $25 \%$ for single junction devices. ${ }^{[8]}$ Even higher efficiencies can be achieved for tandem cells, in which two different light absorbing materials are used with a high bandgap top subcell and low bandgap bottom subcell. This tandem architecture reduces thermalisation losses after photoexcitation of charge carriers and allows the ShockleyQueisser limit on efficiency of single junction cells to be surpassed. Tandem devices combining high bandgap perovskite with a silicon bottom cell have now reached certified efficiency of $29.15 \%,{ }^{[9]}$ significantly higher than for either material in a single junction cell. ${ }^{[10]}$

All-perovskite tandem cells, in which the silicon bottom subcell is replaced by a perovskite absorber with suitably low bandgap, are an even more attractive prospect, offering high efficiencies with reduced manufacturing and environmental costs for power generation. ${ }^{[1-13]}$ The large parameter space of perovskite compositions enables fine control over the bandgap of both top and bottom absorber layers in all-perovskite tandems, ${ }^{[14-16]}$ further reducing energy losses and leading to highly efficient photovoltaic devices. In contrast to silicon, both perovskite absorbers can be processed at low temperatures from widely available precursor materials. Power conversion efficiencies of all-perovskite tandems have recently reached $24.8 \%$ in 2 -terminal cells, ${ }^{[17]}$ and $25 \%$ in 4 -terminal cells,,${ }^{[18]}$ representing large improvements in efficiency over the five years since the first all-perovskite tandems were reported with 


\section{WILEY-VCH}

efficiencies below $11 \%{ }^{[19,20]}$ The choice of low bandgap perovskite is a major factor influencing the performance of all-perovskite tandems. Mixed tin-lead perovskites are an attractive choice, displaying bandgap bowing with tin content which allows tuning of the bandgap to below that of either the neat lead or neat tin compositions. ${ }^{[14,21-24]}$ Further desirable properties of tin-lead perovskites include high charge-carrier mobilities ${ }^{[25-27]}$ and sufficient carrier lifetimes. ${ }^{[27]}$ The first tandem cells to implement mixed tin-lead perovskites as the low bandgap absorber achieved PCE of $17 \% \%^{[28]}$ in a 2-terminal configuration, and over $19 \%{ }^{[28,29]}$ in a 4 -terminal configuration. Tin-lead perovskites have since become established as the main choice for the bottom subcell in all-perovskite tandems, ${ }^{[17,18,30,31]}$ and careful control over the properties of these materials is therefore crucial for achieving further gains in efficiency.

A key challenge of working with tin-containing perovskites is the ready oxidation of $\mathrm{Sn}^{2+}$ to $\mathrm{Sn}^{4+}$, which leads to tin vacancy defects and hole doping in the perovskite semiconductor and is a major degradation pathway. ${ }^{[32-34]}$ Alloying tin with lead has been found to increase the stability of the perovskite, particularly for lead content of at least $50 \%,{ }^{[33,35]}$ so that compositions for low bandgap cells may be chosen to limit tin content in the interests of stability. ${ }^{[36]}$ However, in many cases the desirable minimum bandgap in tin-lead perovskites occurs for majority tin compositions, ${ }^{[28,29,26,37]}$ and limiting tin content to improve stability is therefore a barrier to exploiting the full range of bandgap tunability. The use of additives to control or compensate for the oxidation of tin and improve performance is therefore ubiquitous. By far the most common additive is $\mathrm{SnF}_{2}$, which has been shown to suppress hole doping owing to oxidation of $\mathrm{Sn}^{2+}$ in neat tin perovskites ${ }^{[38-40]}$ and is widely used in tin-lead perovskites for this purpose. ${ }^{[28,35,36,41-45]}$ Other additives including ascorbic acid, ${ }^{[46]}$ GuaSCN, ${ }^{[18]} \mathrm{Cd}^{2+},{ }^{[47]} \mathrm{ZnI}_{2},{ }^{[48]}$ and metallic tin, ${ }^{[17]}$ have also been used, many of these in combination with $\mathrm{SnF}_{2}{ }^{[17,18,47,48]}$ 


\section{WILEY-VCH}

Despite $\mathrm{SnF}_{2}$ being so widely used, its effects on mixed tin-lead perovskites have not previously received comprehensive investigation. Studies on neat-tin perovskites with no alloying of metal cations have explored the influence of $\mathrm{SnF}_{2}$ on optoelectronic and structural properties of these materials, ${ }^{[49,50]}$ and compared the effects of 0,5 , and $10 \mathrm{~mol} \%$ addition of $\mathrm{SnF}_{2 .}{ }^{[40]}$ However, these investigations did not consider mixed tin-lead compositions. Other studies have tried different amounts of $\mathrm{SnF}_{2}$ to optimise solar cell performance for mixed tinlead perovskites, ${ }^{[51-53]}$ but these have neither formed a consensus as to the amount of $\mathrm{SnF}_{2}$ needed, nor examined in depth the underlying changes in properties of the perovskite active layer upon addition of $\mathrm{SnF}_{2}$ which contribute to performance. In addition, optimisation of $\mathrm{SnF}_{2}$ content for performance of tin-lead perovskites has been reported only for single A cation compositions, ${ }^{[51-53]}$ although in tandem cell applications mixed A cations are much more common in the low-bandgap perovskite and have offered higher efficiencies to date. ${ }^{[29]}$ In most cases, tin-lead perovskites for low bandgap cells or subcells in tandem devices are prepared with $10 \% \mathrm{SnF}_{2}$ added, ${ }^{[35,36,44-48,54,55]}$ regardless of tin fraction and often without providing a reason for the choice. While $10 \% \mathrm{SnF}_{2}$ has been established as an effective choice for neat tin perovskites, ${ }^{[40]}$ which may have contributed to this amount being widely used, a determination of optimum amounts of $\mathrm{SnF}_{2}$ for tin-lead perovskites is lacking. This leaves a clear need to identify the amount of $\mathrm{SnF}_{2}$ additive that would effectively prevent tin oxidation in mixed tin-lead perovskites with varying tin content, and to more fully understand the impact of $\mathrm{SnF}_{2}$ on these materials.

Here, we identify the effects of $\mathrm{SnF}_{2}$ addition on the optoelectronic and structural properties of $\mathrm{FA}_{0.83} \mathrm{Cs}_{0.17} \mathrm{Sn}_{x} \mathrm{~Pb}_{1-x} \mathrm{I}_{3}$ perovskite thin films, for tin fraction $x$ varying across the full range from 0 to 1 . The choice of mixed FACs cations at the A site offers improved stability to heat, oxygen, light, and moisture ${ }^{[35,56]}$ and has been used in tandem cells exceeding $23 \%$ 


\section{WILEY-VCH}

efficiency. ${ }^{[36]}$ To identify the amount of $\mathrm{SnF}_{2}$ additive required to reduce oxidation of tin, and investigate any potential unwanted effects from adding larger amounts, we examine films prepared with $0,0.1,1,5,10$, or $20 \mathrm{~mol}^{\circ} \mathrm{SnF}_{2}$ added to the tin precursor solution. This approach ensures that the percentage of $\mathrm{SnF}_{2}$ for each condition is consistent relative to tin content across the range of tin fractions $x$ from 0.1 to 1 . We note that this methodology implies that lead-only compositions (with $x=0$ ) have no $\mathrm{SnF}_{2}$ added. The range of $\mathrm{SnF}_{2}$ percentages we explore here encompasses commonly used amounts ${ }^{[44-48,52-55]}$ as well as smaller quantities of the additive than have usually been considered, e.g. 0.1 and $1 \%$, which we still find to have significant effects on material properties.

We determine from $\mathrm{x}$-ray diffraction measurements that perovskite thin films with tin fraction greater than 0.5 and no $\mathrm{SnF}_{2}$ added display significant tetragonal distortion, attributable to strain caused by tin vacancies and alleviated by addition of $\mathrm{SnF}_{2}$. Dark terahertz conductivity measurements reveal an increase in the frequency of the optical phonon mode associated with vibrations of the metal-halide sublattice ${ }^{[57]}$ as tin fraction increases, confirming the previously hypothesised shift when metal cations of lower atomic mass are introduced. ${ }^{[40]}$ Increasing tin fraction is also accompanied by significant broadening of the phonon peak, related to decreasing phonon lifetimes. Background hole densities, calculated from terahertz conductivity spectra, are significantly reduced by addition of $1 \% \mathrm{SnF}_{2}$ with the largest effect seen for high tin fractions. Photoluminescence lifetimes determined from time-correlated single photon counting measurements are similarly enhanced by addition of $1 \% \mathrm{SnF}_{2}$. Larger amounts of $\mathrm{SnF}_{2}$ give only limited further reduction in hole doping and smaller improvements in photoluminescence lifetime compared to compositions with no additive, suggesting a detrimental effect of excess $\mathrm{SnF}_{2}$ addition. We find that the bandgap bowing trend in absorption onset is distorted by Burstein-Moss shifts in the absorption at high tin fraction when less than $1 \% \mathrm{SnF}_{2}$ is added during preparation, whereas redshifts in emission at low tin 


\section{WILEY-VCH}

fractions are unaffected by $\mathrm{SnF}_{2}$ addition. Broadening of photoluminescence spectra accompanying this redshift demonstrates increased energetic disorder with the introduction of small amounts of tin, which may be mitigated by the addition of moderate amounts of $\mathrm{SnF}_{2}$. Finally, we demonstrate that the increase in charge-carrier mobility upon reduction of background doping follows the same trend as previously observed in inorganic semiconductors. ${ }^{[58]}$ This finding implies that the previously reported low charge-carrier mobilities in tin-based perovskites ${ }^{[59]}$ are predominantly limited by carriers scattering off charged dopants, which can be efficiently reduced by the addition of $\mathrm{SnF}_{2}$ even in small quantities, as we show in this work.

\section{Results and Discussion}

We first consider how structural features are affected by varying amounts of $\mathrm{SnF}_{2}$ additive in $\mathrm{FA}_{0.83} \mathrm{Cs}_{0.17} \mathrm{Sn}_{\mathrm{x}} \mathrm{Pb}_{1-\mathrm{x}} \mathrm{I}_{3}$ thin films. In Section 2.1, we examine the crystal structure of the perovskite lattice through X-ray diffraction, which is followed in Section 2.2 by an exploration of the vibrational modes of the lattice using terahertz dark conductivity spectra. Section 2.3 then describes the impact of $\mathrm{SnF}_{2}$ additive on the background hole doping density, which informs the discussion of its effect on optoelectronic properties further below. In Section 2.4 we identify the $\mathrm{SnF}_{2}$ quantity that optimizes photoluminescence lifetimes, while Section 2.5 explores how variations in $\mathrm{SnF}_{2}$ affect absorption onsets, broadening of emission spectra, and Stokes shifts, across the lead-tin compositional range. Finally, in Section 2.6 we demonstrate how $\mathrm{SnF}_{2}$ addition changes the charge-carrier mobility, which is affected by scattering from background hole doping densities.

\subsection{Crystal Structure}

We begin our investigation of the effects of the $\mathrm{SnF}_{2}$ additive by examining the crystal structure of $\mathrm{FA}_{0.83} \mathrm{Cs}_{0.17} \mathrm{Sn}_{x} \mathrm{~Pb}_{1-x} \mathrm{I}_{3}$ thin films with varying tin fraction and $\mathrm{SnF}_{2}$ percentage. The presence of well-defined, sharp perovskite peaks in the measured x-ray diffraction (XRD) 


\section{WILEY-VCH}

patterns shows that all samples are highly crystalline. There are few detectable impurities, as confirmed by comparison of measured data against the XRD patterns for likely impurities (see Section S2.1 of the SI). The crystal structure of $\mathrm{FA}_{0.83} \mathrm{Cs}_{0.17} \mathrm{Sn}_{x} \mathrm{~Pb}_{1-x} \mathrm{I}_{3}$ is generally welldescribed as pseudocubic, showing good agreement between observed and calculated diffraction peak positions for a pseudocubic structure as detailed in Section S2.2 of the SI. However, we observe significant tetragonal lattice distortion in samples with moderate to high tin content and no $\mathrm{SnF}_{2}$ added, which is suppressed on addition of $20 \% \mathrm{SnF}_{2}$. We also find that lattice constants decrease with higher $\mathrm{SnF}_{2}$ content and higher tin fraction, as indicated by diffraction peaks shifting to higher angle, which we associate with the smaller size of tin ions compared to lead in the perovskite structure.

Figure 1 shows the influence of perovskite composition on three of the highest intensity XRD peaks measured, which illustrate the observed structural changes clearly. The analysed peaks are the (100) and (111) peaks of the pseudocubic structure, and the tetragonal (231) peak. In Figure $1 \mathrm{~A}$ these diffraction peaks are shown with dark grey lines for the $0 \% \mathrm{SnF}_{2}$ condition and coloured lines for compositions with $20 \% \mathrm{SnF}_{2}$ added, where the colours match tin fraction $x$ as labelled. The (100) peak to which the plotted data are normalised is typically the strongest perovskite peak present. However, for many samples with tin fraction $x \geq 0.8$ the (231) peak dominates and hence is separately normalised in Figure 1A. These occurrences are indicated in the figure by asterisks marking any peak stronger than the (100) peak in the measured diffraction pattern for a given composition. Notably, the (231) peak is absent for the lowest tin fractions, and grows in intensity as tin content increases, with this trend being particularly pronounced for the samples with no $\mathrm{SnF}_{2}$ added. Integrated intensities for each of these peaks of interest, shown in Figure S5 in the SI, confirm that the trend of increasing (231) peak intensity with tin fraction holds even after accounting for broadening of diffraction peaks. 

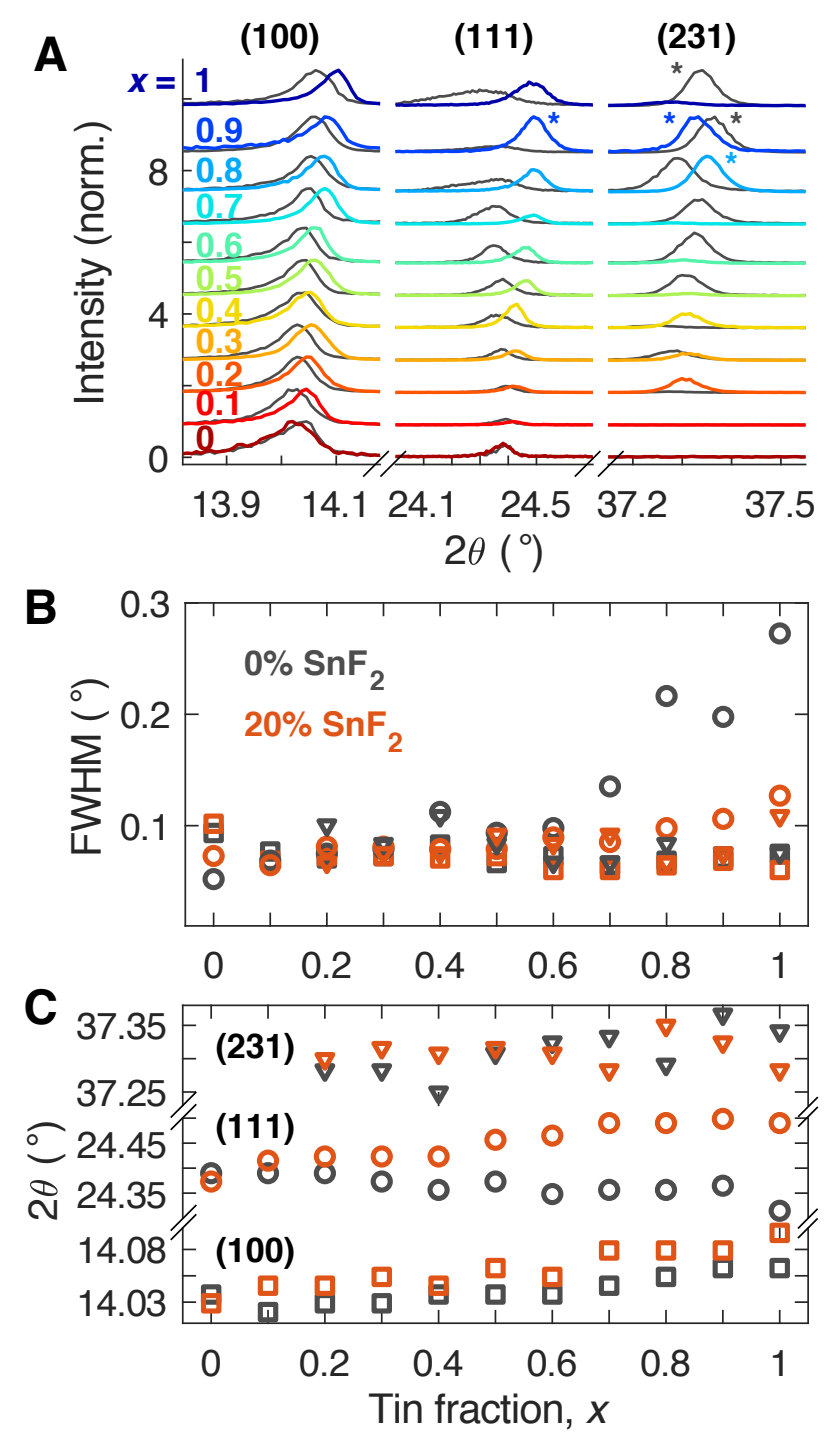

Figure 1. Effect of tin fraction and $\mathrm{SnF}_{2}$ additive on crystal structure of $\mathrm{FA}_{0.83} \mathrm{Cs}_{0.17} \mathrm{Sn}_{x} \mathrm{~Pb}_{1-x} \mathrm{I}_{3}$. Perovskite thin films on quartz substrates were prepared with varying tin fraction and either $0 \%$ or $20 \% \mathrm{SnF}_{2}$ added to the tin precursor solution. XRD patterns were measured with the $\mathrm{Cu}-\mathrm{K}_{\alpha}$ line as incident radiation, and data are shown here for three peaks of interest: pseudocubic (100) and (111), and tetragonal (231), as marked. (A) Normalised XRD patterns, showing three significant peaks for varying tin fractions as marked on the figure. At each tin fraction, data for the $0 \% \mathrm{SnF}_{2}$ condition are shown in dark grey, and data for the $20 \% \mathrm{SnF}_{2}$ condition are overlaid in colour. Vertical offsets have been added for clarity, and in all cases normalisation is to the peak near $14^{\circ}$ (cubic (100)). The peak intensities near $24.4^{\circ}$ and $37.3^{\circ}$ are preserved relative to the $14^{\circ}$ peak, except where marked with $*$, in which case the higher angle peaks are more intense and have been separately normalised for this figure. (B, C) FWHM and position of the XRD peaks shown in panel A, showing significant impact of $\mathrm{SnF}_{2}$ addition on the (111) peak in particular. Data for $0 \% \mathrm{SnF}_{2}$ are shown in dark grey, and for $20 \% \mathrm{SnF}_{2}$ in bright orange, as indicated in panel B. Square markers correspond to the (100) peak, circle markers to the (111) peak, and triangle markers to the tetragonal (231) peak, as indicated in panel $\mathrm{C}$. 


\section{WILEY-VCH}

Broadening of XRD peaks is particularly evident for the pseudocubic (111) peak in Figure

1A. We note that for compositions without $\mathrm{SnF}_{2}$ additive, this peak becomes much broader at high tin fractions. In Figure 1B, broadening is quantified as the full width at half maximum (FWHM) for the three peaks of interest. The pseudocubic (100) peak (data shown by open square markers) and the tetragonal (231) peak (data shown by open downward-pointing triangle markers) both display similar FWHM values across the full range of tin fractions. This is the case independent of whether $0 \%$ (shown in dark grey) or $20 \%$ (shown in orange) $\mathrm{SnF}_{2}$ was added to the tin precursor solution. Similar broadening across the compositional range for both peaks suggests that there is little variation in structural disorder with either tin fraction or $\mathrm{SnF}_{2}$ addition. The broadening of the (111) peak is similarly unchanged for tin fractions from $0-0.6$, but at higher tin fractions the FWHM of this peak dramatically increases when there is no $\mathrm{SnF}_{2}$ added, whereas with addition of $20 \% \mathrm{SnF}_{2}$ there is only a very slight increase in broadening for tin fractions 0.9 and 1.

We determine that the apparent anomalous broadening of the (111) diffraction peak is in fact caused by peak splitting as a consequence of tetragonal distortion of the crystal lattice in samples with high tin to lead ratio. This explanation is suggested by the appearance of the tetragonal (231) peak coinciding with broadening of the (111) peak. Comparing the theoretical diffraction patterns for cubic and tetragonal structure (see Figure S2 in the SI) confirms that a particularly large peak splitting is expected to occur for the (111) peak with tetragonal distortion. Such splitting will give the appearance of broadening as separate peaks cannot be resolved within the measurement limit.

We attribute the observed distortion away from a pseudocubic crystal structure evidenced by these changes in the XRD pattern to the formation of tin vacancies in the lattice following 


\section{WILEY-VCH}

oxidation of $\mathrm{Sn}^{2+} \cdot{ }^{[41,60]}$ The presence of vacancies in the lattice can induce strain which results in distortion towards a lower symmetry structure, as has previously been observed in leadbased perovskites when vacancies are introduced along with intentional doping. ${ }^{[61,62]}$ In the absence of $\mathrm{SnF}_{2}$ additive, samples with higher tin fraction have a higher density of tin vacancies, which results in greater lattice distortion as evidenced by the increasing strength of the tetragonal (231) peak and greater broadening of the pseudocubic (111) peak. The addition of $20 \% \mathrm{SnF}_{2}$ during preparation suppresses the oxidation of tin, reducing background hole doping and eliminating many of the tin vacancies, which explains the dominance of the less distorted pseudocubic structure with $20 \% \mathrm{SnF}_{2}$ addition. Similarly, for low tin fractions there can be relatively few tin vacancies given that most metal sites are occupied by either $\mathrm{Pb}^{2+}$ or $\mathrm{Sn}^{2+}$, in agreement with the evident lack of significant tetragonal distortion even when no $\mathrm{SnF}_{2}$ had been added.

The diffraction peak positions shown in Figure 1C illustrate a further trend of peak shifts to higher angle with increasing tin fraction. Similar shifts in the position of diffraction peaks have previously been reported for a range of tin-lead iodide perovskites in the literature. ${ }^{[37,21,63]}$ This trend results from lattice contraction when $\mathrm{Pb}^{2+}$ is replaced by $\mathrm{Sn}^{2+}$, which has a smaller ionic radius. However, we further note that the addition of $\mathrm{SnF}_{2}$ also shifts the observed pseudocubic diffraction peaks to higher angle, most apparent in Figure 1 for the (100) peak. We attribute this trend to greater propensity for $\mathrm{Sn}^{2+}$ ions to be incorporated into the lattice upon $\mathrm{SnF}_{2}$ addition which may result in a higher stoichiometric tin fraction than originally intended, in particular when as large an amount as $20 \% \mathrm{SnF}_{2}$ is added. This shift to higher angle is also seen for the (111) peak with $20 \% \mathrm{SnF}_{2}$ addition, whereas in the absence of the additive this peak appears to shift slightly to lower angle for high tin fractions in contradiction of the general trend. This anomalous behaviour in the (111) peak position is consistent with tetragonal distortion causing partial splitting of the peak 


\section{WILEY-VCH}

which obscures the shift to higher angle, and so provides further evidence for the role of $\mathrm{SnF}_{2}$ in reducing lattice strain and distortion arising from tin vacancies.

Through this analysis of prominent peaks in the XRD pattern of $\mathrm{FA}_{0.83} \mathrm{Cs}_{0.17} \mathrm{Sn}_{x} \mathrm{~Pb}_{1-x} \mathrm{I}_{3}$ thin films, we have identified that the $\mathrm{SnF}_{2}$ additive has a significant influence on the crystal structure of the perovskite, by preventing formation of tin vacancies and associated lattice strain. We demonstrate that the intensity of the tetragonal (231) peak, and the width of the pseudocubic (111) peak are strong indicators of tetragonal distortion, which can therefore be used to effectively monitor material quality for tin vacancies.

\subsection{Optical Phonon Response}

Following our characterisation of the crystal structure of $\mathrm{FA}_{0.83} \mathrm{Cs}_{0.17} \mathrm{Sn}_{x} \mathrm{~Pb}_{1-x} \mathrm{I}_{3}$ thin films, we next examine how the vibrational modes of the perovskite crystal lattice change with tin fraction. To achieve this, we measure terahertz transmission spectra through samples in the dark (without photoexcitation), for $\mathrm{FA}_{0.83} \mathrm{Cs}_{0.17} \mathrm{Sn}_{x} \mathrm{~Pb}_{1-x} \mathrm{I}_{3}$ films with varying tin fraction $x$, and with $20 \% \mathrm{SnF}_{2}$ added to the tin precursor solution during the deposition process (see Section 4 below and Section S1.1 of the SI for details). Figure 2A shows the real part of terahertz dark conductivity spectra measured in this way. We note that terahertz dark conductivity generally comprises the contribution from free charge carriers as well as the lattice response of the material. However, we find that for samples with $20 \% \mathrm{SnF}_{2}$ added, the free charge-carrier response is suppressed owing to the reduction in background hole doping density, therefore exposing optical phonon modes of the perovskite lattice vibrations. Focusing on the optical phonon peak near $1 \mathrm{THz}$, previously assigned to vibration of the metal-halide sublattice in lead iodide perovskites, ${ }^{[57]}$ we observe an increase in frequency as the tin fraction $x$ increases 


\section{WILEY-VCH}

from 0 to 1 , which we attribute to the lower mass of tin compared to lead in the perovskite structure.
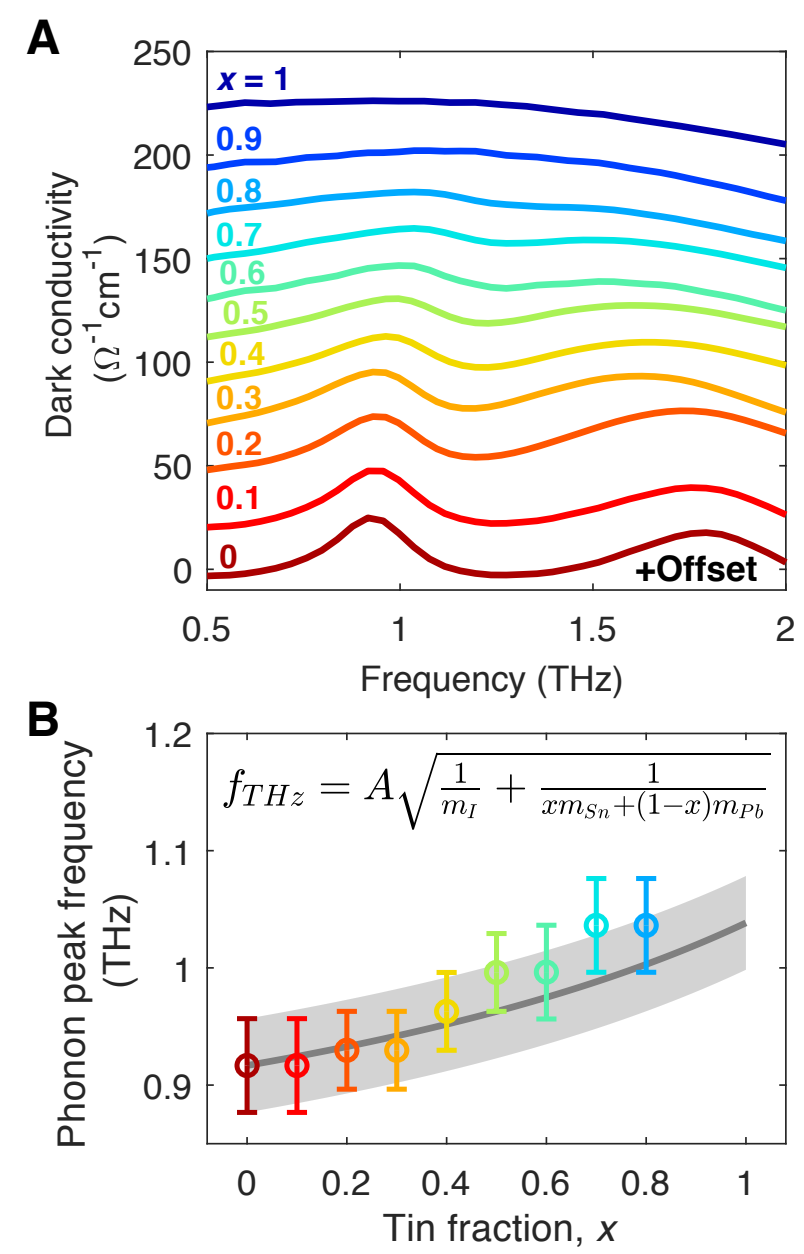

Figure 2. Dark conductivity spectra (panel A) and trend in frequency of the optical phonon peak near $0.8-1 \mathrm{THz}$ across the lead to tin range (panel B) of $\mathrm{FA}_{0.83} \mathrm{Cs}_{0.17} \mathrm{Sn}_{x} \mathrm{~Pb}_{1-x} \mathrm{I}_{3}$. Perovskite thin films on z-cut quartz were prepared with $20 \% \mathrm{SnF}_{2}$ added to the tin precursor solution. (A) Dark conductivity spectra (real part) of samples at tin fractions $x$ as indicated, with vertical offsets of $+20 \Omega^{-1} \mathrm{~cm}^{-1}$ per sample added for clarity, showing the broadening and shift in frequency of the optical phonon peak as tin fraction increases. (B) Relationship between tin fraction and position of the $1 \mathrm{THz}$ optical phonon peak, associated with vibration of the lead-iodide and tin-iodide sublattices. The error bars shown for peak positions represent the spectral resolution of the measurement. The grey solid line shows frequencies for varying tin fraction predicted from the $x=0$ phonon peak using the equation shown for a simple relationship between frequency and reduced mass of the inorganic elements. The uncertainty of these predicted values is shown by the shaded grey region. Frequency data for $x=0.9$ or 1 are not shown, since these spectra are too broadened to allow clear identification of the peak positions.

To confirm the origin of this shift, we plot the frequency of the $\sim 1 \mathrm{THz}$ phonon peak against the tin fraction $x$ in Figure 2B. We then consider a simple model of masses on a spring, for which the frequency of vibrations scales with the reduced mass of the ions involved, 


\section{WILEY-VCH}

according to the formula shown in the figure. The grey line shows the predicted phonon frequency, obtained by extrapolating the phonon peak position at $x=0$ to higher ratios of tin, according to the expression shown. The shaded area represents the uncertainty of this prediction, originating from the spectral resolution of the measurement (shown as error bars for each data point). The data points for tin fractions 0.9 and 1 were omitted in the figure, as the broadening of the conductivity spectrum (which we discuss further below) does not allow for an accurate determination of the phonon peak position.

Within the resolution of the measurement, the data in Figure 2B for tin fractions 0.1 and above agree well with the prediction, indicating that the frequency shift of the phonon peak can be sufficiently explained by the average decrease of the mass of the metal ion in the perovskite structure for tin fraction $x$ increasing from 0 to 1 . We note that this model is highly simplistic, as it does not account for many other factors that might influence phonon frequencies, including changes in local ordering of lead and tin, crystal structure and chemical bonding across the lead-tin compositions. However, the relatively good agreement between the simple model and the optical phonon frequency suggests that for this mode, changes in effective mass may be the dominant factor. We also note that this shift of the optical phonon peak has not been observed previously in tin-based perovskites due to significant spectral broadening, which, together with the background hole doping contribution to the dark conductivity spectrum, obscures the phonon modes in neat tin $(x=1)$ perovskites. The reduction of background doping density achieved here through $\mathrm{SnF}_{2}$ addition was therefore necessary to allow a definitive observation of the frequency shift with gradually increasing tin fraction.

The broadening of the dark conductivity spectrum, associated with a shortening of the optical phonon lifetime, ${ }^{[64]}$ can be partially attributed to the decrease of mass of the metal ion in the 


\section{WILEY-VCH}

perovskite structure, as indicated by theoretical calculations of optical phonon lifetimes in inorganic semiconductors. ${ }^{[65,66]}$ As these optical phonon modes have previously been shown to contribute significantly to the thermal conductivity of metal-halide perovskites, ${ }^{[67]}$ we propose that the shorter phonon lifetimes we find here could be directly responsible for the lower thermal conductivity ${ }^{[68]}$ of tin-based perovskites (compared to their lead-based analogues) that have been reported in the literature. ${ }^{[69,70]}$ In combination with control over electrical conductivity through $\mathrm{SnF}_{2}$ addition, thermal conductivity adjustment by varying the tin:lead ratio could therefore be useful in thermoelectric applications. Our observations therefore offer a clear pathway towards tuning the thermoelectric figure of merit in metal-halide perovskites. $^{[69]}$

\subsection{Background Doping Density}

We are further able to determine the background doping density for $\mathrm{FA}_{0.83} \mathrm{Cs}_{0.17} \mathrm{Sn}_{x} \mathrm{~Pb}_{1-x} \mathrm{I}_{3}$ films as a function of $\mathrm{SnF}_{2}$ addition from dark $\mathrm{THz}$ conductivity spectra. As discussed above, for high background doping densities resulting from hole doping induced by tin vacancies, these spectra are dominated by the response of free charge carriers, rather than by vibrational modes of the lattice. This is illustrated in Figure 3A, where the real part of the terahertz dark conductivity measured for thin films of $\mathrm{FA}_{0.83} \mathrm{Cs}_{0.17} \mathrm{SnI}_{3}$ perovskite $(x=1)$ is shown to be spectrally flat, indicating significant Drude-like response from free charge carriers with short scattering time. ${ }^{[40]}$ We note that the value of the dark conductivity is high when no tin fluoride is added during fabrication, and drops significantly upon the addition of $0.1 \%$ or $1 \%$ of $\mathrm{SnF}_{2}$ to the tin precursor solution during deposition, with less dramatic further decrease seen for larger $\mathrm{SnF}_{2}$ content. To quantify the changes in background hole density responsible for the electrical conductivity of the films, we first separate out the influence of the phonon response on the terahertz transmission spectra. The approach used to accomplish this is described in 


\section{WILEY-VCH}

Section S4 of the SI, where Figure S7 illustrates how the phonon response contribution is subtracted from the measured dark conductivity spectra such as those shown in Figure 3A. We then estimate the background hole doping densities caused by tin oxidation from the measured values of the free carrier dark conductivity and the charge-carrier mobility, obtained from Optical-Pump-THz-Probe (OPTP) measurements (described further in Section 4 below).

Calculated values of the hole doping density are shown in Figure 3B, clearly illustrating the reduction in background doping with tin fluoride addition, indicated by the decrease of dark conductivity shown in panel A. The doping densities calculated here are comparable to previously reported values at the $100 \%$ tin end of the range. ${ }^{[38,40,71]}$ We note that the observed reduction in background doping does not scale linearly with the concentration of $\mathrm{SnF}_{2}$ additive and that even small amounts of tin fluoride in the tin precursor solution $(0.1 \%$ and $1 \%)$ are very effective at suppressing tin oxidation in thin films, with further increases of the additive not influencing the background hole doping as significantly. We also find that for a given percentage of $\mathrm{SnF}_{2}$ additive, background doping density generally increases superlinearly with tin fraction, reflecting a greater tendency towards oxidation of tin in samples with higher tin fraction. As a consequence of this, addition of tin fluoride has limited effect in $\mathrm{FA}_{0.83} \mathrm{Cs}_{0.17} \mathrm{Sn}_{x} \mathrm{~Pb}_{1-x} \mathrm{I}_{3}$ thin films with tin fraction $x \leq 0.6$ where oxidation is already mostly suppressed by means of lead substitution. For $\mathrm{FA}_{0.83} \mathrm{Cs}_{0.17} \mathrm{Sn}_{x} \mathrm{~Pb}_{1-x} \mathrm{I}_{3}$ films with $x=0.6$ the measured dark conductivity spectra are dominated by the phonon response, and differences between spectra with varying $\mathrm{SnF}_{2}$ percentages are similar to the uncertainty of the measurement (see Figure S7 in the SI). Overall, our findings demonstrate that for tin fractions of 0.6 and above, $\mathrm{SnF}_{2}$ is effective at suppressing tin oxidation with just $1 \%$ addition to the tin precursor solution, and that the gains from adding higher percentages of tin fluoride in terms of reducing doping density are modest. For lower tin fractions $(x \leq 0.6,) \mathrm{SnF}_{2}$ is 


\section{WILEY-VCH}

relatively obsolete, given that lead substitution appears to suppress tin vacancy formation and the associated dark hole conductivities more effectively.

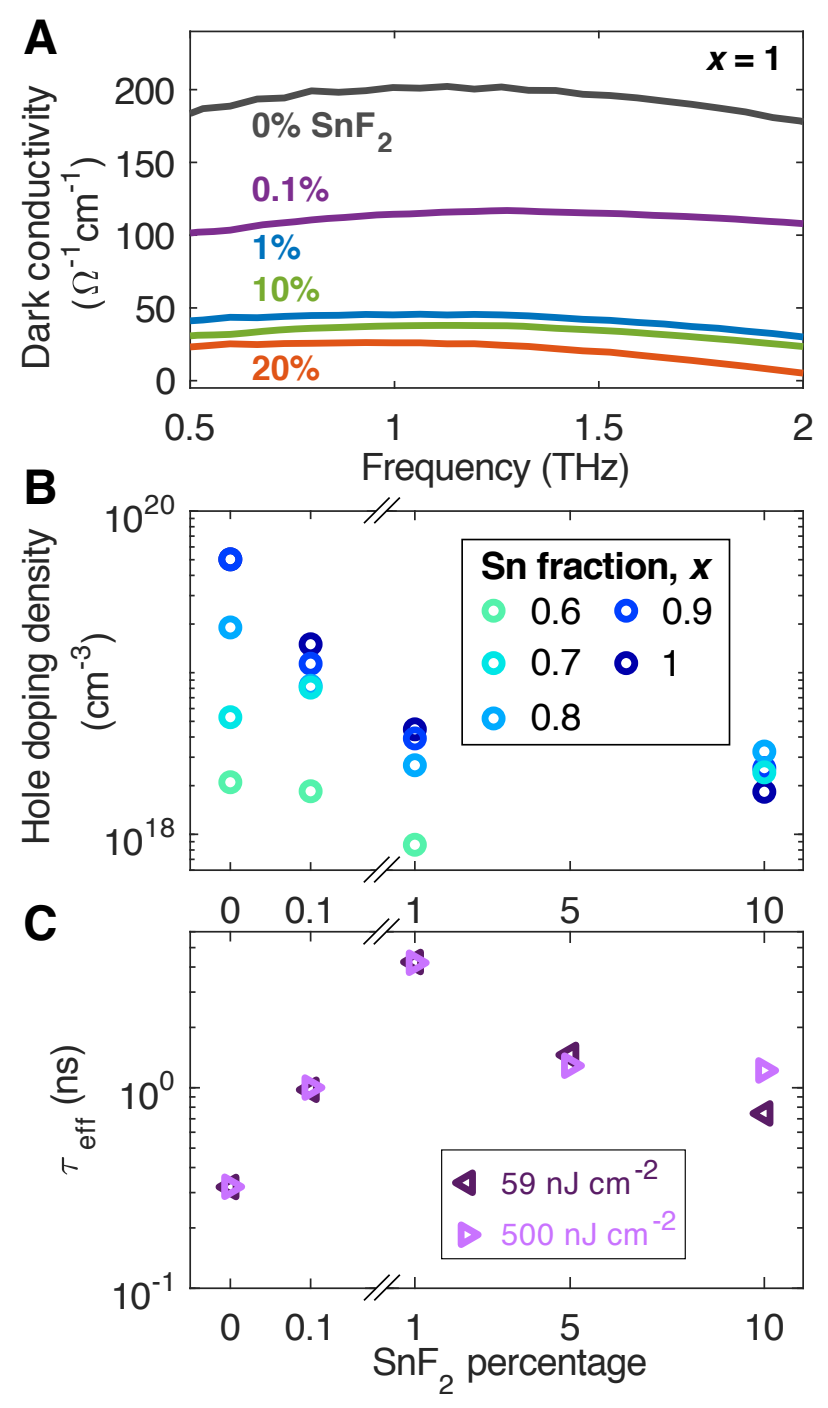

Figure 3. Effect of different amounts of $\mathrm{SnF}_{2}$ additive on the dark conductivity (panel $\mathrm{A}$ ), the corresponding background hole density (panel B), and effective photoluminescence lifetime (panel C) of $\mathrm{FA}_{0.83} \mathrm{Cs}_{0.17} \mathrm{Sn}_{x} \mathrm{~Pb}_{1-x} \mathrm{I}_{3}$ thin films on quartz with tin fraction $x$ between 0.6 and 1 . (A) Dark conductivity spectra (real part) of neat tin $(x=1)$ samples prepared with varying amounts of $\mathrm{SnF}_{2}$ added to the tin precursor solution during preparation as indicated. The drop in conductivity as $\mathrm{SnF}_{2}$ content increases is indicative of reduced background hole doping. (B) Background hole densities determined from dark conductivity and charge-carrier mobility measurements. Samples with $5 \% \mathrm{SnF}_{2}$ added were not used in the measurement. (C) Mean PL lifetime for compositions with tin fraction $0.6-1$, for samples excited at $405 \mathrm{~nm}$ with fluences as indicated. Effective lifetimes were determined by stretched exponential fits to measured PL decay transients.

\subsection{Photoluminescence Lifetimes}




\section{WILEY-VCH}

We further explore how the addition of $\mathrm{SnF}_{2}$ affects charge carrier lifetimes across the $\mathrm{FA}_{0.83} \mathrm{Cs}_{0.17} \mathrm{Sn}_{x} \mathrm{~Pb}_{1-x} \mathrm{I}_{3}$ series. As Figure $3 \mathrm{C}$ illustrates, the addition of $\mathrm{SnF}_{2}$ and associated reduction in background hole doping density leads to an increase in photoluminescence lifetimes. The data shown here represent averages over the lifetimes recorded for films with tin fraction in the range $0.6-1$, at each given tin fluoride percentage. This subset of samples was chosen for comparability of the PL lifetimes with the background hole densities shown in panel B of this figure, which were calculated for the same set of samples. The effective lifetime for each sample was determined from a stretched exponential fit to the measured PL decay transient, as described in Section S5 of the SI. For both low and high excitation fluences, shown by left- and right-pointing open triangle markers in panel C, adding any amount of $\mathrm{SnF}_{2}$ leads to an increase in the lifetime of PL decay compared to samples with $0 \%$ $\mathrm{SnF}_{2}$, with the largest effect seen for $1 \% \mathrm{SnF}_{2}$. The slower PL decay seen on addition of $\mathrm{SnF}_{2}$ can be attributed to the reduction in density of background holes and associated pseudomonomolecular recombination pathways. ${ }^{[40,72,23]}$ The longer PL lifetimes for $1 \% \mathrm{SnF}_{2}$ compared with $0.1 \%$ are then also explained by the difference in background hole doping density between these two conditions. However, a small reduction in lifetime with $5 \%$ or $10 \%$ $\mathrm{SnF}_{2}$ addition relative to $1 \% \mathrm{SnF}_{2}$ is evident in Figure 3C, despite such samples exhibiting similar or slightly lower background hole densities to those with $1 \% \mathrm{SnF}_{2}$. Such subtle decrease of PL lifetimes therefore suggests that the addition of larger amounts of tin fluoride may result in the introduction of other defects, such as formation of $\mathrm{SnF}_{2}$ aggregates, ${ }^{[73]}$ which may create additional trap-assisted recombination pathways, even while recombination with background holes is reduced. The introduction of such pathways would increase the rate of non-radiative recombination, which, like recombination with background holes, is monomolecular. 


\section{WILEY-VCH}

With regards to changes with tin content $x$ across the $\mathrm{FA}_{0.83} \mathrm{Cs}_{0.17} \mathrm{Sn}_{x} \mathrm{~Pb}_{1-x} \mathrm{I}_{3}$ series, we note that PL lifetimes generally decrease with increasing tin fraction (see Section S5 in the SI), as observed previously, ${ }^{[23]}$ and resulting from increasing background hole doping (Figure 3B). For high tin fractions PL decay transients appear monoexponential, whereas for low tin fractions the decay profile is non-exponential (see Figure S8-S10 in the SI), reflecting the dominance of radiative pseudo-monomolecular recombination at high tin fractions which have high background doping. ${ }^{[23]}$ This dominance of recombination with background holes for perovskites with high tin fraction is to be expected for the initial photoexcited charge-carrier densities on the order of $10^{15}-10^{17} \mathrm{~cm}^{-3}$ in our TCSPC measurements, which are lower than the calculated hole doping densities of $>10^{18} \mathrm{~cm}^{-3}$. Addition of $\mathrm{SnF}_{2}$ extends the range of compositions which show non-exponential decay profiles to higher tin fractions as a consequence of the reduction in doping density, with more pronounced beneficial impact for $1 \% \mathrm{SnF}_{2}$ than $5 \%$ or $10 \%$. This reflects the role of $\mathrm{SnF}_{2}$ addition in reducing pseudomonomolecular recombination with background holes, but also reaffirms that, when added in larger amounts, the additive leads to a creation of additional non-radiative recombination centers.

\subsection{Changes in Absorption and Emission Properties}

We now consider how fundamental properties such as absorption onsets and emission spectra of $\mathrm{FA}_{0.83} \mathrm{Cs}_{0.17} \mathrm{Sn}_{x} \mathrm{~Pb}_{1-x} \mathrm{I}_{3}$ are affected by the variations in $\mathrm{SnF}_{2}$ addition and background hole doping. Figure 4 shows the changes in absorption onset (panel A), PL peak width (panel B), and Stokes shift (panel C) for films with varying $\mathrm{SnF}_{2}$ percentage at each tin fraction across the range.

\subsubsection{Absorption Onset}




\section{WILEY-VCH}

Figure 4A shows the variation in absorption onset across the range from lead to tin at different $\mathrm{SnF}_{2}$ percentages, with $E_{g}$ determined from the absorption edge of the sample using the steepest gradient method as described in Section S6.1 of the SI. The change in $E_{g}$ with tin fraction is not linear, showing a minimum for $x=0.6-0.7$ with the value for neat tin and neat lead compositions both higher in energy than most of the intermediate compositions. This trend is known as bandgap bowing, which has previously been observed in several studies of tin-lead perovskites. ${ }^{[14,21-24]}$ For $\mathrm{SnF}_{2}$ content varying from $1 \%$ to $20 \%$, we find little variation in the observed values of $E_{g}$, and the same bowing trend is seen. However, with 0 or $0.1 \%$ $\mathrm{SnF}_{2}$ added to the tin precursor solution (shown in dark grey and purple respectively in the figure) we observe a steeper increase in $E_{g}$ from its minimum value to the neat tin endpoint ( $x$ $=1$ ) than for other $\mathrm{SnF}_{2}$ percentages. Fitting the data for $E_{g}$ against tin fraction $x$ with the parabolic model $E_{g}(x)=E_{S n} x+E_{P b}(1-x)-b x(1-x)$ commonly used to describe bandgap bowing ${ }^{[74-76]}$ further highlights this change in the bandgap at high tin content, as shown in Figure S13 in the SI, and further discussed in Section S6.2 of the SI. For samples with $1-20 \% \mathrm{SnF}_{2}$ added, the values we determine for the bowing parameter $b$ (which describes deviation from linearity) are in agreement with previous reports on closely related perovskite compositions. ${ }^{[23,24]}$ By contrast, for samples with 0 or $0.1 \% \mathrm{SnF}_{2}$ added, fits to the data are poor and return larger $b$ values, and bowing minima are found to occur at lower tin fraction (Figure S13 in the SI). 


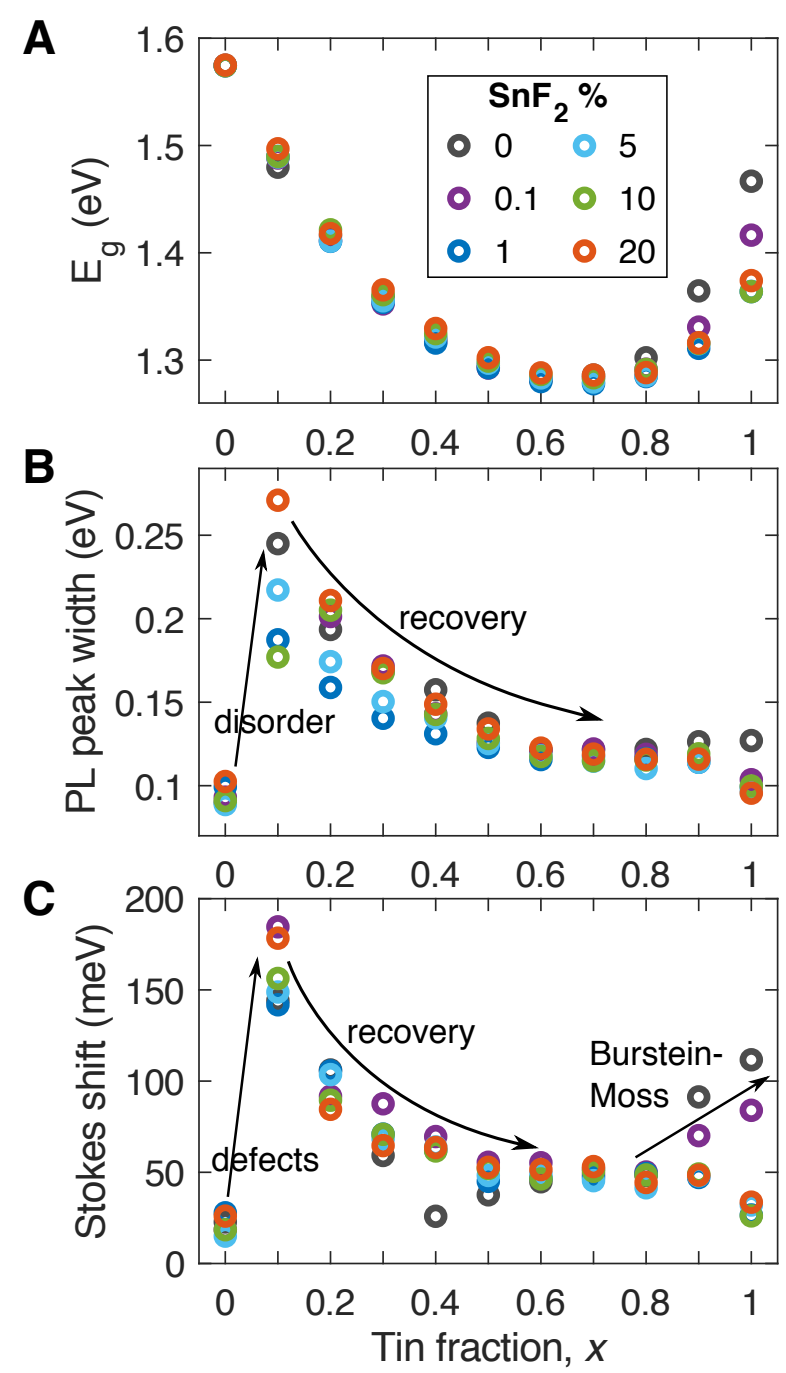

Figure 4. Effect of tin fluoride additive on absorption onset, PL broadening and Stokes shift for $\mathrm{FA}_{0.83} \mathrm{Cs}_{0.17} \mathrm{Sn}_{x} \mathrm{~Pb}_{1-x} \mathrm{I}_{3}$ thin film samples with tin fraction $x$ ranging from 0 to 1 . (A) Variation in the absorption onset energy $\mathrm{E}_{\mathrm{g}}$, showing deviation from the expected trend of bandgap bowing across the range from neat lead to neat tin when little or no $\mathrm{SnF}_{2}$ is added due to the Burstein-Moss effect, with the expected trend restored on addition of $1 \% \mathrm{SnF}_{2}$ or greater. The colour scheme used for different $\mathrm{SnF}_{2}$ percentages shown in the legend is also used for panels B and C. (B) Full width at half maximum of observed PL peaks showing the sharp increase in disorder for low tin compositions, which is improved by the addition of moderate amounts of tin fluoride. PL spectra were measured with CW excitation at $532 \mathrm{~nm}$, and an intensity of $1.8 \pm 0.1 \mathrm{~W} \mathrm{~cm}^{-2}$. (C) Variation in Stokes shift, showing the impact of defects giving more red-shifted emission for low tin fraction, and larger Stokes shift for high tin compositions due to the Burstein-Moss effect, suppressed with addition of $1 \% \mathrm{SnF}_{2}$ or greater.

From the fits of the bandgap bowing model to data, we conclude that the increase in bandgap at high tin fractions and low $\mathrm{SnF}_{2}$ additive level is a deviation from the expected parabolic bandgap bowing behaviour. We find that the addition of at least $1 \% \mathrm{SnF}_{2}$ restores the 


\section{WILEY-VCH}

expected trend in bandgap for the alloying of metals. We attribute this behaviour to the high background hole doping densities present in samples with 0 or $0.1 \% \mathrm{SnF}_{2}$ at high tin fraction, as shown in Figure 3B. These high densities of background holes lower the Fermi level of the semiconductor to the extent that the valence band maximum is mostly empty of electrons (which could be equivalently considered as being filled with holes). Consequently, the highest energy states from which an electron can be photoexcited into the conduction band are found further below the valence band maximum than would be expected for an undoped semiconductor. This situation results in a blueshift in the absorption onset known as the Burstein-Moss shift, ${ }^{[77,78]}$ which has previously been observed in tin perovskites. ${ }^{[40,79]} \mathrm{We}$ observe that such behaviour is clearly discernible across high tin content $(x>0.8)$ lead-tin perovskites, and show that even the addition of as little as $1 \% \mathrm{SnF}_{2}$ is sufficient to eliminate any significant change in absorption onset arising from such Burstein-Moss effects. These observations highlight that the presence of tin vacancies and the associated background hole doping need to be taken into account when choosing tin-lead perovskite composition for use in two-terminal tandem cells, which rely on careful photocurrent matching. We further illustrate that the addition of as little as $1 \% \mathrm{SnF}_{2}$ can eliminate such complicating BursteinMoss shifts in the absorption.

\subsubsection{Photoluminescence Peak Broadening}

We next investigate the impact of $\mathrm{SnF}_{2}$ addition on the shape of the emission spectra of tinlead perovskites with varying tin fraction. We focus first on the observed broadening of the PL peaks, which displays significant variation across the range of tin fractions as well as some dependence on $\mathrm{SnF}_{2}$ percentage at the low-tin end of the compositional range. Figure 4B shows the full width at half maximum of measured PL peaks, quantifying these changes in the emission broadening. In contrast to the observed changes in absorption spectra, the largest variations of PL peak broadening are evident for samples with low tin fraction, between $x=$ 


\section{WILEY-VCH}

0.1 - 0.4. A sharp increase in the width of the PL peaks occurs between $x=0$ and $x=0.1$, with further increases in tin fraction yielding a gradual reduction in peak width to only slightly larger values than those for the neat lead composition.

We attribute this large emission broadening for low-tin compositions to disorder caused upon introduction of small amounts of tin, consistent with the recent identification of an electronically defective compositional region for tin fractions $x=0.05-0.2 \cdot{ }^{[80]}$ No corresponding broadening was apparent in the XRD patterns, as shown in Figure 1, which indicates that the crystallinity of the samples is not significantly affected on addition of small amounts of tin, neither is there structural distortion which would broaden the XRD peaks. The disorder effects upon introduction of tin into a predominantly lead perovskite film apparent in Figure 4B must therefore primarily originate from a broadening of the energetic distribution of electronic states, as discussed further below.

In addition, Figure 4B shows that emission peak widths vary with the quantity of $\mathrm{SnF}_{2}$ added. For $\mathrm{FA}_{0.83} \mathrm{Cs}_{0.17} \mathrm{Sn}_{x} \mathrm{~Pb}_{1-x} \mathrm{I}_{3}$ with $x=1$ (neat tin) we observe a reduction in broadening upon $\mathrm{SnF}_{2}$ addition (compared to no additive being used), which is also present for low tin fractions $x=0.1-0.4$ when moderate amounts of $\mathrm{SnF}_{2}$ are added. We attribute this decrease in broadening to a reduction in energetic disorder, following the suppression of tin-vacancy formation upon $\mathrm{SnF}_{2}$ addition. Such vacancies will likely accept electrons upon release of holes to the valance band, leading to a local variation in electric fields that will translate into an energetically varying landscape for charge carriers. However, for low tin fractions $x=0.1$

-0.4 , this effect appears to be present only for low $\mathrm{SnF}_{2}$ percentages, with excess $\mathrm{SnF}_{2}$ leading to an increased broadening, most likely because additional energetic disorder is introduced upon $\mathrm{SnF}_{2}$ aggregation which overcompensates for the beneficial effects deriving from the reduction in background hole density. 


\section{WILEY-VCH}

\subsubsection{Stokes Shift}

We further examine the Stokes shifts between absorption onset and PL peak energy, shown in Figure $4 \mathrm{C}$, for $\mathrm{FA}_{0.83} \mathrm{Cs}_{0.17} \mathrm{Sn}_{x} \mathrm{~Pb}_{1-x} \mathrm{I}_{3}$. For low tin fractions, a similar trend to that seen in the PL broadening appears, with a large, sudden change in the Stokes shift observed between tin fractions 0 and 0.1 , followed by a gradual recovery as tin fraction increases. The sudden change in Stokes shift is mostly caused by a large redshift in the PL peak energy for low tin content (shown in Figure S16 and S17 in the SI) rather than changes in the absorption onset, which follow a smooth bandgap bowing trend (Figure 4A). Similar redshifts in PL at low tin fraction have previously been reported for mixed lead-tin perovskites incorporating a range of A cations. ${ }^{[23,81,82]}$ These observations can be attributed to the presence of sub-bandgap defect states, which has also been evidenced by large Urbach energies indicating high defect density. ${ }^{[26,83]}$ As already mentioned above, our samples show no noticeable increase in broadening of XRD peaks for low tin fractions compared to the rest of the compositional range (Figure 1B). We therefore consider it unlikely that the observed defectiveness for low tin $\mathrm{FA}_{0.83} \mathrm{Cs}_{0.17} \mathrm{Sn}_{x} \mathrm{~Pb}_{1-x} \mathrm{I}_{3}$ originates from large scale structural disorder or a change in the phase of the perovskite, but rather, it is likely caused by energetic disorder. We also note that there is no trend in the Stokes shift with $\mathrm{SnF}_{2}$ percentage for the low tin fraction samples, and we do not find any impurity peaks in the XRD patterns for these low tin fraction samples (see Figure $\mathrm{S} 1$ and Section $\mathrm{S} 2.1$ of the SI), so excess $\mathrm{SnF}_{2}$ or the presence of other impurities are unlikely to be responsible for introducing these defects. We therefore propose that the addition of small fractions of tin instead leads to changes in the nature and energies of trap states, ${ }^{[23]}$ resulting in an enhancement of sub-bandgap states which red-shifts and broadens the emission substantially. 


\section{WILEY-VCH}

While the observed Stokes shifts decline with increasing tin fractions beyond $x=0.1$, they significantly rise again for $x>0.8$, when less than $1 \% \mathrm{SnF}_{2}$ is added during preparation and tin oxidation is not sufficiently supressed (Figure 4C). The increase in Stokes shift here is caused by the Burstein-Moss shift affecting the absorption onset for samples with high background hole doping concentrations, as discussed above and shown in panel A of Figure 4. Since during the PL emission process, photoexcited electrons can first relax down to the conduction band edge, and then radiatively recombine with either free photoexcited holes or background holes occupying states near the valence band edge, we do not expect a BursteinMoss shift to occur in emission spectra. Indeed, as can be seen in Figure S17 in the SI, no change of PL peak energy occurs with different contents of $\mathrm{SnF}_{2}$, even for the high tin fractions. Therefore, the observed Stokes shifts for highly doped tin-rich perovskites are simply attributable to the Burstein-Moss effect.

The Stokes shift thus reflects two key trends in the optoelectronic properties of $\mathrm{FA}_{0.83} \mathrm{Cs}_{0.17} \mathrm{Sn}_{x} \mathrm{~Pb}_{1-x} \mathrm{I}_{3}$ perovskite: defectiveness and associated energetic disorder of perovskite films at low tin fraction, and the influence of hole doping resulting from oxidation of tin which causes a Burstein-Moss shift in the absorption onset at high tin fractions. Both of these phenomena are important to consider when assessing tin-lead perovskites for use in lowbandgap solar cells or tandem devices. The poor material quality at low tin fractions makes these compositions less desirable to use, while the influence of hole doping which is particularly apparent at high tin fractions can shift absorption onsets, unless compensated with the addition of at least $1 \%$ relative $\mathrm{SnF}_{2}$ content.

\subsection{Charge-Carrier Mobility}




\section{WILEY-VCH}

We further explore how charge transport properties of tin-lead iodide perovskites are affected by $\mathrm{SnF}_{2}$ addition, given that charge-carrier mobilities in the active layer of a solar cell play a crucial role in determining its performance. To obtain high photocurrent under solar illumination, photoexcited charge carriers have to be efficiently extracted from the absorber material. In devices with planar geometry this can be achieved by ensuring that the chargecarrier diffusion length (which depends on the mobility and lifetime of the photoexcited charge carriers) exceeds the thickness of the active layer. ${ }^{[84]}$ Although in general chargecarrier diffusion lengths achievable in hybrid perovskites were shown to be high, ${ }^{[85]}$ for tinbased perovskites, the reduction of PL lifetime and charge-carrier mobility leads to a shortening of diffusion lengths and a decrease in device performance when compared with their lead-based counterparts. ${ }^{[40,59,86]}$ We therefore conclude our investigations by examining the charge-carrier mobility in thin films of $\mathrm{FA}_{0.83} \mathrm{Cs}_{0.17} \mathrm{Sn}_{x} \mathrm{~Pb}_{1-x} \mathrm{I}_{3}$ perovskites and its relation to hole doping concentration in these materials, controlled by the addition of $\mathrm{SnF}_{2}$ to the tin precursor solution. Figure 5 shows the sum electron-hole mobilities determined from OPTP measurements (see Section S8 of the SI) for thin-films with tin fraction 0.6 or greater, at different $\mathrm{SnF}_{2}$ addition and therefore different hole background doping concentrations. As noted in Section 2.3 and illustrated in Figure S7, the differences between dark conductivity spectra of samples with varying $\mathrm{SnF}_{2}$ percentage approach the uncertainty of the measurement for samples with tin fraction $x=0.6$ as the phonon response contribution becomes dominant. Perovskites with $x<0.6$, for which the dominance of the phonon response in dark conductivity spectra would prohibit reliable determination of charge-carrier density, are therefore not considered here. We report a significant increase of charge-carrier mobility as background doping is reduced in perovskites with high tin fraction. However, we note that the addition of $\mathrm{SnF}_{2}$ to thin films with lower tin ratio $(x \leq 0.7)$ does not change the mobility of the charge carriers significantly, in accordance with the already low presence of hole doping in these materials. 


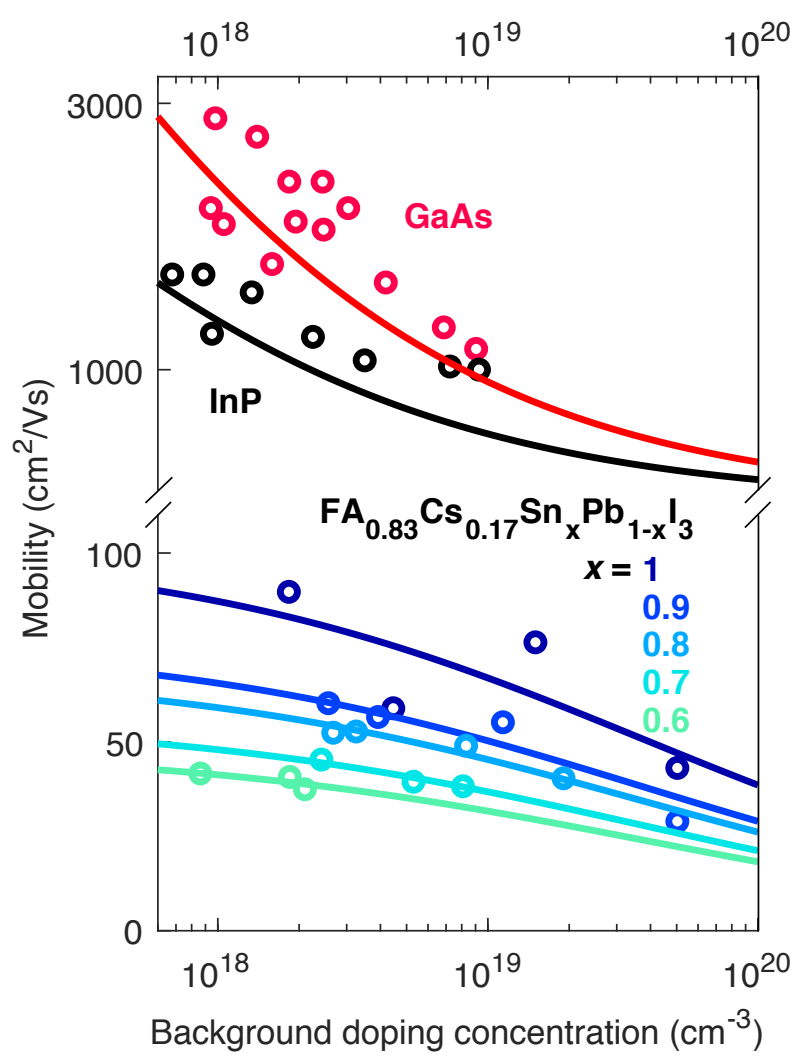

Figure 5. Decrease in mobility with increasing levels of background doping for $\mathrm{FA}_{0.83} \mathrm{Cs}_{0.17} \mathrm{Sn}_{x} \mathrm{~Pb}_{1-x} \mathrm{I}_{3}$ at varying tin fractions, compared to two inorganic semiconductors. Data for GaAs and InP were extracted from Hilsum, C. Simple empirical relationship between mobility and carrier concentration Electronics Letters, 10, 13, 1974, 259-260, and the fits shown as red and black solid lines are the same as in that study. Fits for the perovskite chargecarrier sum mobilities using the empirical formula by Hilsum (with normalising density of $N_{0}$ $=3.76 \times 10^{19}$ optimised for all samples, and $\mu_{\mathrm{L}}$ values as shown in Table S1 in the SI) were obtained using the least squares method and are shown by solid lines following the colour scheme shown for tin fraction $x$.

To explore the origin of these mobility changes, we follow the approach by Hilsum, ${ }^{[58]}$ previously applied to doped inorganic semiconductors such as GaAs and InP. The model assumes that the charge-carrier mobility in doped semiconductors is limited by lattice scattering (electron-phonon coupling) and impurity scattering of the carriers, and that only the impurity scattering rate is dependent on the dopant concentration, with the lattice scattering rate at room temperature being an intrinsic property of the material. ${ }^{[58]}$ The Hilsum formula relates the overall charge-carrier mobility $\mu$ to the dopant concentration $N$ by:

$\mu=\frac{\mu_{L}}{1+\sqrt{\frac{N}{N_{0}}}}$ 


\section{WILEY-VCH}

where $\mu_{\mathrm{L}}$ is the lattice-scattering-limited value of the charge-carrier mobility and $N_{0}$ is a normalising density of dopants, determined empirically.

Figure 5 shows the relation between charge-carrier mobility and background doping density together with fits of Equation 1, contrasting our results for $\mathrm{FA}_{0.83} \mathrm{Cs}_{0.17} \mathrm{Sn}_{x} \mathrm{~Pb}_{1-x} \mathrm{I}_{3}$ thin films from OPTP measurements with those reported previously by Hilsum ${ }^{[58]}$ for two inorganic semiconductors, GaAs and InP. Fitting parameters are provided in Table S6 in the SI. Our analysis reveals that trends in the charge-carrier mobilities of tin-lead iodide perovskite are consistent with the prediction that scattering off dopants limits charge-carrier mobilities in these materials (similarly to inorganic semiconductors). We also report that for significantly reduced background dopant density, the charge-carrier mobility increases appreciably as the tin fraction in the crystal structure is increased. Such behaviour is consistent with the calculated lower charge-carrier effective masses in tin-based perovskites, ${ }^{[87]}$ and underlines that once the background dopant density is reduced in tin-halide perovskites, their intrinsic material properties begin to dominate. These findings therefore highlight the importance of reducing background hole density in order to improve the optoelectronic properties of mixed tin-lead perovskites for PV applications, particularly for compositions involving high tin fraction.

\section{Conclusion}

In conclusion, we have unravelled the effects of the commonly used $\mathrm{SnF}_{2}$ additive on $\mathrm{FA}_{0.83} \mathrm{Cs}_{0.17} \mathrm{Sn}_{x} \mathrm{~Pb}_{1-x} \mathrm{I}_{3}$ perovskites across the compositional range from lead to tin. We compare the effects of $\mathrm{SnF}_{2}$ addition at percentages spanning three orders of magnitude, to identify how much of this additive is needed to realise the intended beneficial effects of reducing tin oxidation in mixed tin-lead perovskites without side effects on material quality 


\section{WILEY-VCH}

from excess $\mathrm{SnF}_{2}$. We find that addition of $1 \% \mathrm{SnF}_{2}$ results in a significant reduction in the background hole density from tin oxidation, leading to longer photoluminescence lifetimes, decreased energetic disorder, reduced Burstein-Moss shift (distorting the bandgap bowing trend in absorption onsets), and higher charge-carrier mobilities. By comparison, adding $0.1 \%$ $\mathrm{SnF}_{2}$ does not fully realise these beneficial effects, while for $5 \% \mathrm{SnF}_{2}$ and above modest additional reductions in background hole density are accompanied by the introduction of defects from excess tin fluoride. These defects increase energetic disorder for low-tin compositions in particular, and enhance non-radiative recombination, which limits photoluminescence lifetimes. Alongside these effects on optoelectronic properties, we report that the addition of $\mathrm{SnF}_{2}$ reduces tetragonal distortion in the perovskite structure, particularly evident at high tin fractions and attributed to the presence of tin vacancies. These point defects cause strain in the crystal structure, which is alleviated by $\mathrm{SnF}_{2}$ addition. For perovskite films with background hole density reduced by $\mathrm{SnF}_{2}$ addition, we are also able to distinguish the optical phonon response associated with inorganic lattice vibrations which demonstrates a shift to higher frequency and significant broadening as tin fraction increases. Our results reveal that many of the benefits of the $\mathrm{SnF}_{2}$ additive for tin and mixed tin-lead perovskites can be realised with smaller amounts than have been commonly used. The impact of $\mathrm{SnF}_{2}$ addition is also found to vary with tin fraction, having limited effects for majority lead compositions while playing a significant role in improving the structural and optoelectronic properties of thin films with higher tin fractions. This work therefore provides a foundation for the improvement of low-bandgap mixed tin-lead perovskite semiconductors through greater understanding of the role played by $\mathrm{SnF}_{2}$ addition across the full compositional range.

\section{Experimental Section}




\section{WILEY-VCH}

Solution preparation. Two parent solutions (of $\mathrm{FA}_{0.83} \mathrm{Cs}_{0.17} \mathrm{PbI}_{3}$ and $\mathrm{FA}_{0.83} \mathrm{Cs}_{0.17} \mathrm{SnI}_{3}$, both 1.2 Molar) were prepared 12 hours before sample fabrication, in the mixed solvent DMF:DMSO (with a volumetric ratio of 4:1). A $3.5 \mathrm{~mL}$ solution with tin fraction $0: \mathrm{FA}_{0.83} \mathrm{Cs}_{0.17} \mathrm{PbI}_{3}$ 599.49mg of Formamidinium Iodide (FAI, Greatcell solar), 185.50mg of Caesium Iodide (CsI, Alfa Aesar) \& 1936.24mg Lead (II) Iodide ( $\mathrm{PbI}_{2}$, Alfa Aesar, 99.999\% mesh beads). A $3 \mathrm{~mL}$ solution with tin fraction 1: $\mathrm{FA}_{0.83} \mathrm{Cs}_{0.17} \mathrm{SnI}_{3}-513.85 \mathrm{mg}$ of FAI, $159.00 \mathrm{mg}$ of CsI, $1341.07 \mathrm{mg}$ of Tin (II) Iodide ( $\mathrm{SnI}_{2}$, Alfa Aesar, 99.999\% mesh beads) \& Tin (II) fluoride $\left(\mathrm{SnF}_{2}\right.$, Sigma Aldrich) corresponding to $0,0.1,1,5,10$ or $20 \mathrm{~mol} \%$ as indicated in the discussion above. These solutions were then mixed in the manner detailed in Table S1 in the SI to give the desired $\mathrm{Sn}$ fraction at chosen $\mathrm{SnF}_{2}$ content. Previous studies have demonstrated that the tin fraction in mixed tin-lead perovskites is well controlled by the stoichiometry of the precursor solution, independent of whether $\mathrm{SnF}_{2}$ additive is used. $[14,21,25,45,88,89]$

Sample fabrication. Z-cut quartz substrates ( $2 \mathrm{~mm}$ thick, $13 \mathrm{~mm}$ diameter discs) were prepared by sonicating sequentially in Decon 90 detergent (1\% vol. in deionised (DI) water), DI water, Acetone and Ethanol for 10 minutes followed by $\mathrm{O}_{2}$ plasma treatment for 15 minutes. Films were prepared on quartz using a single step anti-solvent quenching method in a nitrogen filled glovebox $\left(\mathrm{H}_{2} \mathrm{O} \leq 0.1 \mathrm{ppm}, \mathrm{O}_{2} \leq 1.0 \mathrm{ppm}\right) .20 \mu \mathrm{L}$ of the required solution was dispensed onto a static substrate and spun at 4000rpm with an acceleration of 500rpm for a total of 60 seconds. At $35 \mathrm{~s} 100 \mu \mathrm{L}$ of anisole was dispensed into the centre of the substrate at a height of roughly $5 \mathrm{~mm}$ from the surface in a steady stream taking around 1 second to fully dispense. For films with tin fraction 0.7 or less the films turned amber when the anisole was introduced getting progressively darker from $x=0-0.7$. For films containing tin fraction 0.8 and above the film turned very dark brown to black instantly. Substrates were then transferred straight onto a hotplate and annealed for 10 minutes at $100{ }^{\circ} \mathrm{C}$ resulting in specular black 


\section{WILEY-VCH}

films across the Sn range. All film preparation parameters were kept constant for each sample. After annealing, all samples were stored in a nitrogen filled glovebox.

$X$-ray diffraction measurement. XRD patterns were obtained for $\mathrm{FA}_{0.83} \mathrm{Cs}_{0.17} \mathrm{Sn}_{x} \mathrm{~Pb}_{1-x} \mathrm{I}_{3}$ thin films on z-cut quartz substrates, as well as for a blank quartz substrate, using a PANalytical X'Pert PRO Powder X-Ray Diffractometer. Each measurement covered diffraction angles $2 \theta$ from $10-54.5$ at a scan speed of $0.0088 \mathrm{~s}^{-1}$, using the $\mathrm{Cu}-\mathrm{K}_{\alpha}$ line at $1.54 \AA$ as incident radiation. Each sample was brought from the glovebox in an individual vacuum sealed bag, minimising exposure to air before starting the XRD measurement, which was conducted under ambient atmosphere. This was the last measurement conducted for any given sample, so that air exposure whilst acquiring the XRD pattern did not influence any other results. To correct for sample tilt, the highest intensity z-cut quartz peak present in all of the measured patterns was used as a reference and set to $2 \theta=50.66^{\circ}$.

Absorbance spectra. Reflectance and transmittance spectra for $\mathrm{FA}_{0.83} \mathrm{Cs}_{0.17} \mathrm{Sn}_{x} \mathrm{~Pb}_{1-x} \mathrm{I}_{3}$ thin films on z-cut quartz substrates were measured using a Bruker Vertex 80v Fourier transform infrared spectrometer with reflection/transmission accessory fitted. Samples were transported under nitrogen, and measurements conducted under low vacuum (pressure below $3 \mathrm{mbar}$ ). A tungsten halogen lamp was used as the source, with $\mathrm{CaF}_{2}$ beamsplitter and Si diode detector. Reference spectra for transmittance and reflectance were measured with the sample position empty, and a UV-protected silver mirror (Thorlabs) in the sample position, respectively. The reference-corrected reflectance and transmittance spectra for each sample were then used to calculate (Napierian) absorbance as $A=-\ln (T /(1-R))$.

Photoluminescence spectra. Samples were loaded into a vacuum cell inside the nitrogen filled glovebox used for storage, and kept under vacuum during photoluminescence (PL) 


\section{WILEY-VCH}

measurements with continuous pumping to maintain a pressure of $1 \times 10^{-2}$ mbar or below. A 532nm continuous wave laser (Ventus, Laser Quantum) was used to excite samples at an intensity of $1.8 \pm 0.1 \mathrm{Wcm}^{-2}$, and PL from the sample was then focussed into a grating monochromator (iHR320, Horiba) through a variable width entry slit, using long-pass filters to reduce background signal from the excitation beam. A liquid nitrogen-cooled silicon charge coupled device (Symphony II, Horiba) and a liquid nitrogen-cooled linear InGaAs array (Symphony II, Horiba) were used to detect the dispersed signal, which was directed via a movable mirror onto each detector in turn. A spectral response correction, using a tungsten filament lamp with known spectrum, was applied to the measured PL spectra. As the range of PL peak energies for varying tin fraction overlaps with the onset of detection for the InGaAs array and the drop off in sensitivity of the Si CCD, data measured using both detectors were combined as described in section S7.1 of the SI to give the final PL spectra presented and analysed in this work.

Time-resolved photoluminescence measurements. Samples were loaded into a vacuum cell inside the nitrogen-filled glovebox used for storage, and kept under vacuum with continuous pumping to maintain a pressure of $1 \times 10^{-2}$ mbar or below while carrying out time-correlated single photon counting (TCSPC) measurements for PL decay. Two different setups (described below) were used for these measurements to allow for the variation in PL lifetimes across the range of tin fractions, with excitation at $405 \mathrm{~nm}$ and two different fluences, using neutral density filters to attenuate laser power for the lower fluence.

In the primary TCSPC setup, pulsed diode laser (PicoQuant, LDH-D-C-405M) with variable repetition rate set at $10 \mathrm{MHz}$, or $5 \mathrm{MHz}$ for the neat lead samples $(x=0)$ which exhibit the slowest PL decay, was used to excite the samples at fluences of $0.50 \pm 0.01 \mu \mathrm{Jcm}^{-2}$ and $59 \pm$ $1 \mathrm{nJcm}{ }^{-2}$ for the high- and low-power conditions respectively. Background from the laser was 


\section{WILEY-VCH}

reduced by the use of a long-pass filter, and PL was collected and coupled into a grating spectrograph (Princeton Instruments, SP-2558), then directed onto a single-photon avalanche diode detector (PDM series from MPD), with timing controlled by a PicoHarp300 TCSPC event timer. The time resolution of this setup, as determined from the full width at half maximum of the instrument response function measured for the laser pulse, was $0.70 \mathrm{~ns}$.

For TCSPC measurements of samples at high tin fraction with very short PL lifetimes, a higher time-resolution setup was used, in which pulsed excitation was generated by a Ti:Sapphire laser (Mai Tai, Spectra Physics) with a repetition rate of $80 \mathrm{MHz}$, tuned to $810 \mathrm{~nm}$ and subsequently doubled in a $\beta$-barium borate $(\mathrm{BBO})$ crystal to $405 \mathrm{~nm}$, resulting in excitation fluences of $0.48 \pm 0.1 \mu \mathrm{Jcm}^{-2}$ and $51 \pm 10 \mathrm{nJcm}^{-2}$ for the high and low power conditions respectively. PL from the sample, with background light from the excitation beam reduced by a long-pass filter, was focussed into a grating monochromator (Triax, Horiba) through a variable-width entry slit, then to a silicon single-photon avalanche diode detector (PDM series from MPD). A gate beam split off from the excitation laser was directed onto a photodiode as the trigger pulse for timing. The time resolution determined from the full width at half maximum of the instrument response, measured by detecting the laser pulse directly at a wavelength of $810 \mathrm{~nm}$, was $0.06 \mathrm{~ns}$.

\section{Terahertz spectroscopy}

The temporal changes of photoconductivity were obtained with an Optical-pump-THz-probe (OPTP) setup, described in previous publications. ${ }^{[6,57,90]}$ The films were photoexcited with 35 fs ultrafast laser pulses originating from an 800nm Ti-Sapphire laser system (Spectra Physics, Mai Tai - Ascend - Spitfire Ti-Sapphire regenerative amplifier, 5kHz repetition rate), frequency doubled to $400 \mathrm{~nm}$ using a BBO crystal. The fluence of the photoexcitation was varied between 1.33 and $22.5 \mu \mathrm{J} \mathrm{cm}^{-2}$ using a variable ND filter wheel. 


\section{WILEY-VCH}

The photoconductivity of the films was calculated using the transmission data of the terahertz pulse probe through the photoexcited samples (see section S3 in the SI). The THz pulse was generated with a spintronic emitter (2nm tungsten, $1.8 \mathrm{~nm} \mathrm{Co}_{40} \mathrm{Fe}_{40} \mathrm{~B}_{20}$, 2nm platinum on quartz substrate $)^{6}$ and the field strength transmitted through the sample was measured using electro-optic sampling in a non-linear crystal (1mm thick (110)-ZnTe). The THz pulse and gate pulse $(800 \mathrm{~nm}, 35 \mathrm{fs}$, originating from the same laser system as the pump beam) were overlapped inside the ZnTe crystal and the change of the polarisation of the gate beam (proportional to THz field strength) was measured using a quarter-wave plate, polarising prism and balanced photodiode detector. The time delay between the pump pulse and $\mathrm{THz}$ probe was controlled using optical delay stages. The pump beam was blocked using an optical chopper for every second $\mathrm{THz}$ pulse, in order to obtain the $\mathrm{THz}$ transmission difference through the sample due to photoexcited charge carriers.

The dark conductivity spectra were acquired with the same laser setup as used for OPTP measurements, without the use of the photoexcitation beam. The time-domain shape of the THz pulse transmitted through the sample was measured by changing the time-delay between the gate pulse and $\mathrm{THz}$ probe using optical delay stages. A bare z-cut quartz substrate was used as a reference. ${ }^{[91]}$ The dark conductivity was calculated from the transmission of the $\mathrm{THz}$ pulse data, as described in Section S4 in the SI.

Both the OPTP and dark conductivity measurements were conducted in a vacuum box under pressure $<10^{-1}$ mbar. The samples were stored in a nitrogen-filled glovebox and transported to the vacuum box in an air-tight bag filled with nitrogen to minimise air exposure. 


\section{WILEY-VCH}

\section{Supporting Information}

Supporting Information is available from the Wiley Online Library or from the author.

\section{Acknowledgements}

The authors acknowledge financial support from the Engineering and Physical Sciences

Research Council (EPSRC), UK. K.J.S. thanks the Rhodes Trust for financial support through a Rhodes Scholarship. A.M.U. thanks the EPSRC Centre for Doctoral Training (CDT) for Science and Applications of Plastic Electronic Materials for financial support through a graduate scholarship.

Received: ((will be filled in by the editorial staff))

Revised: ((will be filled in by the editorial staff)) Published online: ((will be filled in by the editorial staff))

References

[1] S. De Wolf, J. Holovsky, S.-J. Moon, P. Löper, B. Niesen, M. Ledinsky, F.-J. Haug, J.-H. Yum, C. Ballif, J. Phys. Chem. Lett. 2014, 5, 1035.

[2] V. D’Innocenzo, G. Grancini, M. J. P. Alcocer, A. R. S. Kandada, S. D. Stranks, M. M. Lee, G. Lanzani, H. J. Snaith, A. Petrozza, Nat. Commun. 2014, 5, 3586.

[3] K. Galkowski, A. Mitioglu, A. Miyata, P. Plochocka, O. Portugall, G. E. Eperon, J. T.W. Wang, T. Stergiopoulos, S. D. Stranks, H. J. Snaith, R. J. Nicholas, Energy Environ. Sci. 2016, $9,962$.

[4] G. Xing, N. Mathews, S. S. Lim, N. Yantara, X. Liu, D. Sabba, M. Grätzel, S. Mhaisalkar, T. C. Sum, Nat. Mater. 2014, 13, 476.

[5] H. Oga, A. Saeki, Y. Ogomi, S. Hayase, S. Seki J. Am. Chem. Soc. 2014, 136, 13818.

[6] C. Wehrenfennig, G. E. Eperon, M. B. Johnston, H. J. Snaith, L. M. Herz, Adv. Mater. 2014, 26, 1584.

[7] C. S. Ponseca Jr, T. J. Savenije, M. Abdellah, K. Zheng, A. Yartsev, T. Pascher, T. Harlang, P. Chabera, T. Pullerits, A. Stepanov., J.-P. Wolf, V. Sundström, J. Am. Chem. Soc. 


\section{WILEY-VCH}

2014, 136, 5189.

[8] E. H. Jung, N. J. Jeon, E. Y. Park, C. S. Moon, T. J. Shin, T.-Y. Yang, J. H. Noh, J. Seo, Nature 2019, 567, 511.

[9] Helmholtz Zentrum Berlin, World Record: Efficiency of perovskite silicon tandem solar cell jumpts to 29.15 per cent, https://www.helmholtzberlin.de/pubbin/news_seite?nid=21020;sprache=en, accessed: June, 2020.

[10] M. A. Green, E. D. Dunlop, J. Hohl-Ebinger, M. Yoshita, N. Kopidakis, A. W. Y. HoBaillie, Prog. Photovoltaics 2020, 28, 3.

[11] T. Leijtens, K. A. Bush, R. Prasanna, M. D. McGehee, Nat. Energy 2018, 3, 828.

[12] Z. Song, A. B. Phillips, I. Celik, G. K. Liyanage, D. Zhao, D. Apul, Y. Yan, M. J. Heben, in 2018 IEEE 7th World Conference on Photovoltaic Energy Conversion (WCPEC)(A Joint Conference of 45th IEEE PVSC, 28th PVSEC \& 34th EU PVSEC), IEEE, 2018, 1134.

[13] I. Celik, A. B. Phillips, Z. Song, Y. Yan, R. J. Ellingson, M. J. Heben, D. Apul, Energy Environ. Sci. 2017, 10, 1874.

[14] F. Hao, C. C. Stoumpos, R. P. H. Chang, M. G. Kanatzidis, J. Am. Chem. Soc. 2014, $136,8094$.

[15] J. H. Noh, S. H. Im, J. H. Heo, T. N. Mandal, S. I. Seok, Nano Lett. 2013, 13, 1764.

[16] E. L. Unger, L. Kegelmann, K. Suchan, D. Sörell, L. Korte, S. Albrecht, J. Mater. Chem. A 2017, 5, 11401.

[17] R. Lin, K. Xiao, Z. Qin, Q. Han, C. Zhang, M. Wei, M. I. Saidaminov, Y. Gao, J. Xu, M. Xiao, A. Li, J. Zhu, E. H. Sargent, H. Tan, Nat. Energy 2019, 4, 864.

[18] J. Tong, Z. Song, D. H. Kim, X. Chen, C. Chen, A. F. Palmstrom, P. F. Ndione, M. O. Reese, S. P. Dunfield, O. G. Reid, J. Liu, F. Zhang, S. P. Harvey, Z. Li, S. T. Christensen, G. Teeter, D. Zhao, M. M. Al-Jassim, M. F. A. M. Van Hest, M. C. Beard, S. E. Shaheen, J. J. Berry, Y. Yan, K. Zhu, Science 2019, 364, 475.

[19] Z. Li, P. P. Boix, G. Xing, K. Fu, S. A. Kulkarni, S. K. Batabyal, W. Xu, A. Cao, T. C. 


\section{WILEY-VCH}

Sum, N. Mathews, L. H. Wong, Nanoscale 2016, 8, 6352.

[20] J. H. Heo, S. H. Im. Adv. Mater. 2016, 28, 5121.

[21] C. Liu, J. Fan, H. Li, C. Zhang, Y. Mai, Sci. Rep. 2016, 6, 35705.

[22] R. Prasanna, A. Gold-Parker, T. Leijtens, B. Conings, A. Babayigit, H.-G. Boyen, M.

F. Toney, M. D. McGehee, J. Am. Chem. Soc. 2017, 139, 11117.

[23] E. S. Parrott, T. Green, R. L. Milot, M. B. Johnston, H. J. Snaith, L. M. Herz, Adv. Funct. Mater. 2018, 28, 1802803.

[24] A. Rajagopal, R. J. Stoddard, H. Hillhouse, A. K.-Y. Jen, J. Mater. Chem. A 2019, 7, 16285.

[25] C. C. Stoumpos, C. D. Malliakas, M. G. Kanatzidis, Inorg. Chem. 2013, 52, 9019.

[26] B. Zhao, N. Abdi-Jalebi, M. Tabachnyk, H. Glass, V. S. Kamboj, W. Nie, A. J.

Pearson, Y. Puttisong, K. C. Gödel, H. E. Beere, D. A. Ritchie, A. D. Mohite, S. E. Dutton, R. H. Friend, A. Sadhanala, Adv. Mater. 2017, 29, 1604744.

[27] D. Zhao, Y. Yu, C. Wang, W. Liao, N. Shrestha, C. R. Grice, A. J. Cimaroli, L. Guan, R. J. Ellingson, K. Zhu, X. Zhao, R.-G. Xiong, Y. Yan, Nat. Energy, 2017, 2, 17018.

[28] G. E. Eperon, T. Leijtens, K. A. Bush, R. Prasanna, T. Green, J. T.-W. Wang, D. P. McMeekin, G. Volonakis, R. L. Milot, R. May, A. Palmstrom, D. J. Slotcavage, R. A. Belisle, J. B. Patel, E. S. Parrott, R. J. Sutton, W. Ma, F. Moghadam, B. Conings, A. Babayigit, H.-G. Boyen, S. Bent, F. Giustino, L. M. Herz, M. B. Johnston, M. D. McGehee, H. J. Snaith, Science 2016, 354, 861.

[29] Z. Yang, A. Rajagopal, C.-C. Chueh, S. B. Jo, B. Liu, T. Zhao, A. K.-Y. Jen, Adv. Mater. 2016, 28, 8990.

[30] C. Wang, Z. Song, C. Li, D. Zhao, Y. Yan, Adv. Funct. Mater. 2019, 29, 1808801.

[31] S. Gu, R. Lin, Q. Han, Y. Gao, H. Tan, J. Zhu Adv. Mater. 2020, 1907392.

[32] Y. Takahashi, H. Hasegawa, Y. Takahashi, T. Inabe, J. Solid State Chem. 2013, 205, 39. 


\section{WILEY-VCH}

[33] T. Leijtens, R. Prasanna, A. Gold-Parker, M. F. Toney, M. D. McGehee, ACS Energy Lett. 2017, 2, 2159.

[34] H. Yao, F. Zhou, Z. Li, Z. Ci, L. Ding, Z. Jin, Adv. Sci. 2020, 7, 1903540.

[35] T. Leijtens, R. Prasanna, K. A. Bush, G. E. Eperon, J. A. Raiford, A. Gold-Parker, E. J. Wolf, S. A. Swifter, C. C. Boyd, H.-P. Wang, M. F. Toney, S. F. Bent, M. D. McGehee, Sustainable Energy Fuels 2018, 2, 2450.

[36] A. F. Palmstrom, G. E. Eperon, T. Leijtens, R. Prasanna, S. N. Habisreutinger, W. Nemeth, E. A. Gaulding, S. P. Dunfield, M. Reese, S. Nanayakkara, T. Moot, J. Werner, J. Liu, B. To, S. T. Christensen, M. D. McGehee, M. F. A. M. van Hest, J. M. Luther, J. J. Berry, D. T. Moore, Joule 2019, 3, 2193.

[37] M. Anaya, J. P. Correa-Baena, G. Lozano, M. Saliba, P. Anguita, B. Roose, A. Abate, U. Steiner, M. Grätzel, M. E. Calvo, A. Hagfeldt, H. Míguez, J. Mater. Chem. A 2016, 4, 11214.

[38] M. H. Kumar, S. Dharani, W. L. Leong, P. P. Boix, R. R. Prabhakar, T. Baikie, C. Shi, H. Ding, R. Ramesh, M. Asta, M. Grätzel, S. G. Mhaisalkar, N. Mathews, Adv. Mater. 2014, 26,7122 .

[39] T. M. Koh, T. Krishnamoorthy, N. Yantara, C. Shi, W. L. Leong, P. P. Boix, A. C. Grimsdale, S. G. Mhaisalkar, N. Mathews, J. Mater. Chem. A 2015, 3, 14996.

[40] R. L. Milot, M. T. Klug, C. L. Davies, Z. Wang, H. Kraus, H. J. Snaith, M. B. Johnston, L. M. Herz, Adv. Mater. 2018, 30, 1804506.

[41] A. Rajagopal, Z. Yang, S. B. Jo, I. L. Braly, P.-W. Liang, H. W. Hillhouse, A. K.-Y. Jen, Adv. Mater. 2017, 29, 1702140.

[42] D. Zhao, C. Wang, Z. Song, Y. Yu, C. Chen, X. Zhao, K. Zhu, Y. Yan, ACS Energy Lett. 2018, 3, 305.

[43] C. Li, Z. Song, D. Zhao, C. Xiao, B. Subedi, N. Shrestha, M. M. Junda, C. Wang, C.S. Jiang, M. Al-Jassim, R. J. Ellingson, N. J. Podraza, K. Zhu, Y. Yan, Adv. Energy 


\section{WILEY-VCH}

Mater. 2019, 9, 1803135.

[44] J. Wang, K. Datta, J. Li, M. A. Verheijen, D. Zhang, M. M. Wienk, R. A. J. Janssen, Adv. Energy Mater. 2020, 2000566.

[45] M. Wei, K. Xiao, G. Walters, R. Lin, Y. Zhao, M. I. Saidaminov, P. Todorović, A. Johnston, Z. Huang, H. Chen, A. Li, J. Zhu, Z. Yang, Y.-K. Wang, A. H. Proppe, S. O.

Kelley, Y. Hou, O. Voznyy, H. Tan, E. H. Sargent, Adv. Mater. 2020, 32, 1907058.

[46] X. Xu, C.-C. Chueh, Z. Yang, A. Rajagopal, J. Xu, S. B. Jo, A. K.-Y. Jen Nano Energy 2017, 34, 392.

[47] Z. Yang, Z. Yu, H. Wei, X. Xiao, Z. Ni, B. Chen, Y. Deng, S. N. Habisreutinger, X. Chen, K. Wang, J. Zhao, P. N. Rudd, J. J. Berry, M. C. Beard. J. Huang, Nat. Commun. 2019, 10 .

[48] A. R. Bowman, M. T. Klug, T. A. S. Doherty, M. D. Farrar, S. P. Senanayak, B. Wenger, G. Divitini, E. P. Booker, Z. Andaji-Garmaroudi, S. Macpherson, E. Ruggeri, H. Sirringhaus, H. J. Snaith, S. D. Stranks, ACS Energy Lett. 2019, 4, 2301.

[49] S. Gupta, D. Cahen, G. Hodes, J. Phys. Chem. C 2018, 122, 13926.

[50] C. Hartmann, S. Gupta, T. Bendikov, X. Kozina, T. Kunze, R. Félix, G. Hodes, R. G. Wilks, D. Cahen, M. Bär, ACS Appl. Mater. Interfaces 2020, 12, 12353.

[51] C.-M. Tsai, H.-P. Wu, S.-T. Chang, C.-F. Huang, C.-H. Wang, S. Narra, Y.-W. Yang, C.-L. Wang, C.-H. Hung, E. W.-G. Diau, ACS Energy Lett. 2016, 1, 1086.

[52] A. Rajagopal, P.-W. Liang, C.-C. Chueh, Z. Yang, A. K.-Y. Jen, ACS Energy Lett. 2017, 2, 2531.

[53] A. M. Igual-Muñoz, J. Avila, P. P. Boix, H. J. Bolink, Solar RRL 2020, 4, 32.

[54] C. Li, Z. S. Wang, H. L. Zhu, D. Zhang, J. Cheng, H. Lin, D. Ouyang, W. C. H. Choy, Adv. Energy Mater. 2018, 8, 1801954.

[55] Y. Yao, F. Lv, L. Luo, L. Liao, G. Wang, D. Liu, C. Xu, G. Zhou, X. Zhao, Q. Song, Solar RRL 2020, 4, 1900396. 


\section{WILEY-VCH}

[56] J. W. Lee, D.-H. Kim, H-S. Kim, S.-W. Seo, S. M. Cho, N.-G. Park, Adv. Energy

Mater. 2015, 5, 1501310

[57] C. Wehrenfennig, M. Liu, H. J. Snaith, M. B. Johnston, L. M. Herz, Energy Environ. Sci. 2014, 7, 2269.

[58] C. Hilsum, Electron. Lett. 1974, 10, 259.

[59] Herz, L. M. ACS Energy Lett. 2017, 2, 1539.

[60] T. Shi, H.-S. Zhang, W. Meng, Q. Teng, M. Liu, X. Yang, Y. Yan, H.-L. Yip, Y.-J. Zhao, J. Mater. Chem. A 2017, 5, 15124.

[61] A. K. Jena, A. Kulkarni, Y. Sanehira, M. Ikegami, T. Miyasaka, Chem. Mater. 2018, 30,6668 .

[62] Y. Zhou, Y. Zhao, Energy Environ. Sci. 2019, 12, 1495.

[63] Y. Zong, N. Wang, L. Zhang, M.-G. Ju, X. C. Zeng, X. W. Sun, Y. Zhou, N. P. Padture, Angew. Chem., Int. Ed. 2017, 56, 12658.

[64] T. Beechem, S. Graham, J. Appl. Phys. 2008, 103, 093507.

[65] P. Klemens, Phys. Rev. 1966, 148, 845.

[66] A. Bhatt, K. Kim, M. Stroscio, J. Appl. Phys. 1994, 76, 3905.

[67] A. Gold-Parker, P. M. Gehring, J. M. Skelton, I. C. Smith, D. Parshall, J. M. Frost, H. I. Karunadasa, A. Walsh, M. F. Toney, Proc. Natl. Acad. Sci. 2018, 115, 11905.

[68] L. D. Whalley, J. M. Skelton, J. M. Frost, A. Walsh, Phys. Rev. B 2016, 94, 220301.

[69] X. Mettan, R. Pisoni, P. Matus, A. Pisoni, J. Jaćimović, B. Náfrádi, M. Spina, D. Pavuna, L. Forró, E. Horváth, J. Phys. Chem. C 2015, 119, 11506.

[70] W. Lee, H. Li, A. B. Wong, D. Zhang, M. Lai, Y. Yu, Q. Kong, E. Lin, J. J. Urban, J. C. Grossman, P. Yang, Proc. Natl. Acad. Sci. 2017, 114, 8693.

[71] M. Xiao, S. Gu, P. Zhu, M. Tang, W. Zhu, R. Lin, C. Chen, W. Xu, T. Yu, J. Zhu, Adv. Optical Mater. 2018, 6, 1700615 


\section{WILEY-VCH}

[72] R. L. Milot, G. E. Eperon, T. Green, H. J. Snaith, M. B. Johnston, L. M. Herz, J. Phys. Chem. Lett. 2016, 7, 4178.

[73] W. Liao, D. Zhao, Y. Yu, C. R. Grice, C. Wang, A. J. Cimardi, P. Schulz, W. Meng,

K. Zhu, R.-G. Xiong, Y. Yan, Adv. Mater. 2016, 28, 9333

[74] M. Cardona, Phys. Rev. 1963, 129, 69.

[75] A. Thompson, J. Woolley, Can. J. Phys. 1967, 45, 255.

[76] I. Vurgaftman, J. R. Meyer, L. R. Ram-Mohan J. Appl. Phys. 2001, 89, 5815.

[77] E. Burstein, Phys. Rev. 1954, 93, 632.

[78] T. S. Moss, Proc. Phys. Soc., Sect. B 1954, 67, 775.

[79] T. Handa, T. Yamada, H. Kubota, S. Ise, Y. Miyamoto, Y. Kanemitsu, J. Phys. Chem. C 2017, 121, 16158.

[80] M. T. Klug, R. L. Milot, J. B. Patel, T. Green, H. C. Sansom, M. D. Farrar, A. J. Ramadan, S. Martani, Z. Wang, B. Wenger, J. M. Ball, L. Langshaw, A. Petrozza, M. B. Johnston, L. M. Herz, H. J. Snaith, Energy Environ. Sci. 2020, 13, 1776

[81] Z. Zhu, N. Li, D. Zhao, L. Wang, A. K.-Y. Jen, Adv. Energy Mater. 2019, 9, 1802774.

[82] Z. Yang, X. Zhang, W. Yang, G. E. Eperon, D. S. Ginger, Chem. Mater. 2020, 32, 2782

[83] B. Subedi, C. Li, M. M. Junda, Z. Song, Y. Yan, N. J. Podraza, J. Chem. Phys. 2020, $152,064705$.

[84] M. B. Johnston, L. M. Herz, Acc. Chem. Res. 2016, 49, 146.

[85] S. D. Stranks, G. E. Eperon, G. Grancini, C. Menelaou, M. J. Alcocer, T. Leijtens, L. M. Herz, A. Petrozza, H. J. Snaith, Science 2013, 342, 341.

[86] N. K. Noel, S. D. Stranks, A. Abate, C. Wehrenfennig, S. Guarnera, A.-A. Haghighirad, A. Sadhanala, G. E. Eperon, S. K. Pathak, M. B. Johnston, A. Petrozza, L. M. Herz, H. J. Snaith, Energy Environ. Sci. 2014, 7, 3061.

[87] J. Even, L. Pedesseau, J.-M. Jancu, C. Katan, Phys. Status Solidi RRL 2014, 8, 31. 


\section{WILEY-VCH}

[88] Y. Ogomi, A. Morita, S. Tsukamoto, T. Saitho, N. Fujikawa, Q. Shen, T. Toyoda, K. Yoshino, S. S. Pandey, T. Ma, S. Hayase, J. Phys. Chem. Lett. 2014, 5, 1004.

[89] W. Liao, D. Zhao, Y. Yu, N. Shrestha, K. Ghimire, C. R. Grice, C. Wang, Y. Xiao, A. J. Cimaroli, R. J. Ellingson, N. J. Podraza, K. Zhu, R. G. Xiong, Y. Yan, J. Am. Chem. Soc. 2016, 138, 12360.

[90] P. Tiwana, P. Parkinson, M. B. Johnston, H. J. Snaith, L. M. Herz, J. Phys. Chem. C 2010, 114, 1365.

[91] C. Davies, J. Patel, C. Xia, L. M. Herz, M. B. Johnston, J. Infrared, Millimeter, Terahertz Waves, 2018, 39, 1236. 


\section{WILEY-VCH}

The impact of $\mathrm{SnF}_{2}$ on $\mathrm{FA}_{0.83} \mathrm{Cs}_{0.17} \mathrm{Sn}_{x} \mathrm{~Pb}_{1-x} \mathrm{I}_{3}$ perovskite thin films is examined for additive amounts varying between $0.1 \%$ and $20 \%$. Structural distortion from lattice strain is reduced by $\mathrm{SnF}_{2}$ addition. Lower background hole doping, longer PL lifetimes, and higher chargecarrier mobilities are observed with as little as $1 \% \mathrm{SnF}_{2}$ added. Larger quantities of the additive introduce defects alongside these beneficial effects.

Photovoltaic devices

K. J. Savill, A. M. Ulatowski, M. D. Farrar, M. B. Johnston, H. J. Snaith, L.M. Herz*

Impact of tin fluoride additive on the properties of mixed tin-lead iodide perovskite semiconductors

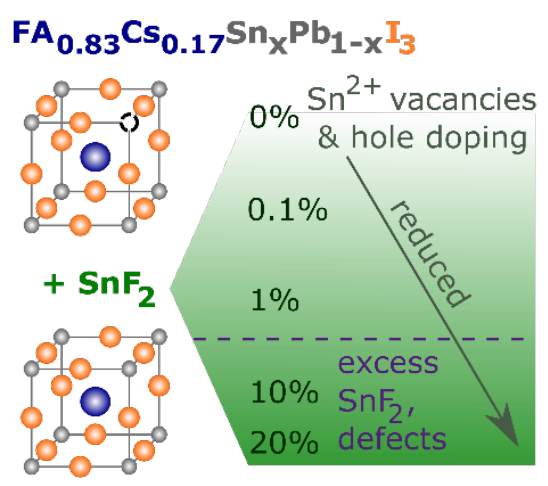

